\title{
Discordant diagnostic criteria for pneumonia in COPD trials: a review
}

\author{
Robert A. Wise $\mathbb{(}^{1}$, Mona Bafadhel $\mathbb{1}^{2}$, Courtney Crim $^{3,18}$, Gerard J. Criner ${ }^{4}$, Nicola C. Day ${ }^{5}$, \\ David M.G. Halpin ${ }^{6}$, MeiLan K. Han ${ }^{7}$, Peter Lange ${ }^{8,9}$, David A. Lipson (10 ${ }^{10,11}$, Fernando J. Martinez ${ }^{12}$, \\ Diego J. Maselli $\mathbb{1}^{13}$, Dawn Midwinter $\mathbb{C}^{5}$, Dave Singh ${ }^{14}$, Maeva Zysman ${ }^{15,16}$, Mark T. Dransfield ${ }^{17}$ and \\ Richard E.K. Russell (10) ${ }^{2}$
}

\begin{abstract}
${ }^{1}$ Division of Pulmonary and Critical Care Medicine, Johns Hopkins University School of Medicine, Baltimore, MD, USA. ${ }^{2}$ Nuffield Dept of Medicine, University of Oxford, Oxford, UK. ${ }^{3}$ Clinical Sciences - Respiratory, GSK, Research Triangle Park, NC, USA. ${ }^{4}$ Lewis Katz School of Medicine at Temple University, Philadelphia, PA, USA. ${ }^{5}$ GlaxoSmithKline, GSK House, Brentford, UK. ${ }^{6}$ University of Exeter Medical School, College of Medicine and Health, University of Exeter, Exeter, UK. ${ }^{7}$ University of Michigan, Pulmonary \& Critical Care, Ann Arbor, MI, USA. ${ }^{8}$ Section of Epidemiology, Dept of Public Health, University of Copenhagen, Copenhagen, Denmark. ${ }^{9}$ Medical Dept, Herlev and Gentofte Hospital, Herlev, Denmark. ${ }^{10}$ Clinical Sciences, GSK, Collegeville, PA, USA. ${ }^{11}$ Pulmonary, Allergy and Critical Care Division, Dept of Medicine, Perelman School of Medicine, University of Pennsylvania, Philadelphia, PA, USA. ${ }^{12}$ New YorkPresbyterian Hospital/Weill Cornell Medical Center, New York, NY, USA. ${ }^{13}$ Dept of Medicine, University of Texas Health at San Antonio, San Antonio, TX, USA. ${ }^{14}$ Centre for Respiratory Medicine and Allergy, Institute of Inflammation and Repair, Manchester Academic Health Science Centre, The University of Manchester, Manchester University NHS Foundation Hospital Trust, Manchester, UK. ${ }^{15}$ Service des Maladies Respiratoires, CHU Bordeaux, Pessac, France. ${ }^{16}$ Univ-Bordeaux, Centre de Recherche cardio-thoracique de Bordeaux, U1045, CIC 1401, Pessac, France. ${ }^{17}$ Division of Pulmonary, Allergy, and Critical Care Medicine, Lung Health Center, University of Alabama at Birmingham, Birmingham, AL, USA. ${ }^{18}$ Affiliation at the time of writing.
\end{abstract}

Corresponding author: Richard Russell (richard.russell@ndm.ox.ac.uk)

Shareable abstract (@ERSpublications)

Trials of ICS in COPD use varying methods of reporting pneumonia incidence, which influence the interpretation of results. A minimal set of standardised criteria for pneumonia diagnosis would allow comparison of pneumonia incidence between trials. https://bit.ly/3nbkdoL

Cite this article as: Wise RA, Bafadhel M, Crim C, et al. Discordant diagnostic criteria for pneumonia in COPD trials: a review. Eur Respir Rev 2021; 30: 210124 [DOI: 10.1183/16000617.0124-2021].

Copyright $\odot$ The authors 2021

This version is distributed under the terms of the Creative Commons Attribution Licence 4.0.

This article has supplementary material available from err.ersjournals.com

Received: 24 May 2021 Accepted: 28 Aug 2021

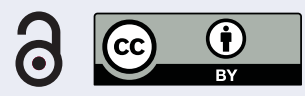

\section{Abstract}

Inhaled corticosteroids (ICS) have a class effect of increasing pneumonia risk in patients with COPD. However, pneumonia incidence varies widely across clinical trials of ICS use in COPD. This review clarifies methodological differences in defining and recording pneumonia events in these trials and discusses factors that could contribute to the varying pneumonia incidence. Literature searches and screening yielded 40 relevant references for inclusion. Methods used to capture pneumonia events in these studies included investigator-reported pneumonia adverse events, standardised list of signs or symptoms, radiographic confirmation of suspected cases and/or confirmation by an independent clinical end-point committee. In general, more stringent pneumonia diagnosis criteria led to lower reported pneumonia incidence rates. In addition, studies varied in design and population characteristics, including exacerbation history and lung function, factors that probably contribute to the varying pneumonia incidence. As such, cross-trial comparisons are problematic. A minimal set of standardised criteria for diagnosis and reporting of pneumonia should be used in COPD studies, as well as reporting of patients' pneumonia history at baseline, to allow comparison of pneumonia rates between trials. Currently, within-trial comparison of ICS-containing versus non-ICS-containing treatments is the appropriate method to assess the influence of ICS on pneumonia incidence.

\section{Introduction}

Community-acquired pneumonia is one of the most common serious infectious diseases, accounting for almost $1 \%$ of all medical admissions [1, 2]. Diagnosis of community-acquired pneumonia using clinical signs and symptoms and laboratory data alone can be inaccurate, due to heterogeneity of clinical presentation, and may be a particular challenge in the presence of chronic respiratory disease [3]. Radiographic confirmation, required to make a definitive diagnosis, is recommended; however, this is often 
not obtained, particularly in the primary care setting [3]. COPD is a known risk factor for community-acquired pneumonia [4-6]. Furthermore, observational studies suggest that exacerbations caused by pneumonia are associated with an increased risk of intensive care unit admission, need for mechanical ventilation, length of stay and mortality compared with exacerbations not caused by pneumonia in patients with COPD [7, 8].

The Global Initiative for Chronic Obstructive Lung Disease strategy report recommends addition of inhaled corticosteroid (ICS) treatment for patients with COPD with persistent exacerbations despite receiving mono or dual long-acting bronchodilator therapy [9]. These recommendations are made on the basis that addition of ICS reduces exacerbation rates and improves lung function and health status in patients with a history of COPD exacerbations [10-15]. However, ICS have a class effect of increasing the risk of pneumonia in patients with COPD [16-19]. Although this class effect is consistently described in the literature, the rates of pneumonia vary between studies and some analyses have described differing pneumonia rates with different ICS therapies [20-22]. In much of the COPD literature it is unclear how pneumonia events are defined, recorded, graded in terms of severity and adjudicated. In addition, methods for pneumonia capture and assessment can differ between countries; for example, computed tomography is reported to be most frequently used in Japan and the United States [23]. Moreover, factors such as the study design, ascertainment of pneumonia events, patient population and characteristics vary between studies. Heterogeneity in the definition of pneumonia is a potential difficulty for meta-analyses of this outcome.

Given the importance of an accurate estimate of the risk of pneumonia with any given COPD therapy when making treatment decisions, we performed an in-depth review of the literature to examine differences in reporting methodologies. Other factors that may contribute to differing rates of pneumonia reporting in COPD clinical trials of ICS therapy were also considered, such as study design and patient population characteristics.

\section{Methods}

We performed literature searches of the PubMed and Embase databases on 10 August 2020, using the search terms "corticosteroid" OR "glucocorticoid” AND "chronic obstructive lung disease" AND ([controlled clinical trial]/lim OR [randomised controlled trial]/lim) AND [2007-2020]/py) AND (“article"/it OR “article in press”/it). Although the search was done some time prior to publication, updating this search to a more recent date would not, in the opinion of the authors, alter the conclusions. Our initial search yielded 749 publications for review, with 458 from PubMed and 291 from EMBASE. The list of trials retrieved was checked by the authors, and one additional relevant study was added as it was not listed in the initial search results [24]. After eliminating duplicates, 615 results remained. The titles, abstracts and full text of these articles were then screened for relevancy, excluding manuscripts corresponding to studies that were $<24$ weeks in length, had a population of $<300$ patients, did not report on patients with COPD or patients receiving ICS-containing therapy, did not compare with a non-ICS-containing treatment, or did not report the incidence of pneumonia (number and/or percentage of patients with pneumonia in each treatment group). Trials included were completed prior to the coronavirus disease 2019 pandemic. Manuscripts focusing on secondary subgroup analyses of clinical trials were also excluded unless they reported the incidence of pneumonia. Following this screening, 40 relevant references remained and were included in the review (figure 1). Each manuscript was examined, and details of the study population and the methodology used to capture pneumonia, as well as the incidence of pneumonia in treatment groups were collected. Risk of pneumonia for each trial was calculated as a fold increase in pneumonia incidence (incidence in treatment arm of interest)/(incidence in comparator arm); however, a limitation of this approach is that not all patients within a study may have been followed for the same length of time. The findings of the analysis are reported in a narrative manner.

Differences in pneumonia capture and reporting

In clinical studies, a trial investigator would typically report adverse events such as pneumonia as per protocol guidance. These events are then coded to an adverse event term using the preferred terms from the Medical Dictionary for Regulatory Activities (MedDRA) prior to analysing the data. As part of these analyses, pneumonia as an adverse event could be reported as a single preferred term, or as part of a group of several pneumonia-related preferred terms, often labelled adverse events of special interest (AESIs). The grouping of MedDRA preferred terms into AESIs is commonplace in randomised controlled trials (RCTs). However, the reliability and reproducibility of the approach has been questioned, as the definition of which preferred terms comprise a pneumonia AESI could differ between trials. For example, some trials may use Standardised MedDRA Queries (validated, pre-determined sets of MedDRA preferred terms grouped together to enable capture of all plausible events linked to a disease process [25]), and some may define 


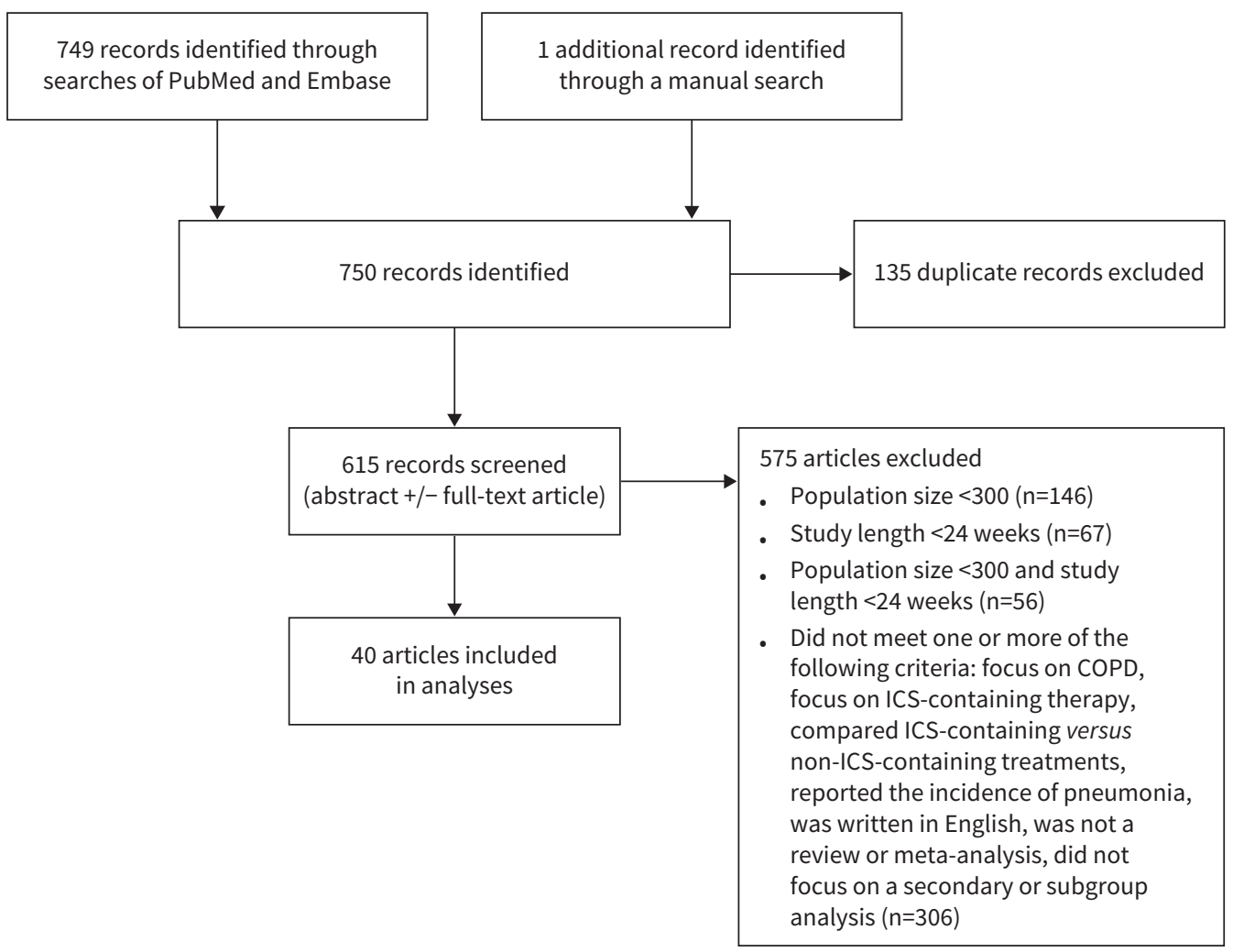

FIGURE 1 Flowchart describing study selection. ICS: inhaled corticosteroid.

their own set of preferred terms (referred to as sponsor-defined AESI throughout the paper). The latter might include only a few or a large number of preferred terms. Regardless of the number of preferred terms in an AESI, it is important to note that investigator-reported pneumonia-related events might not map to all preferred terms within the AESI. Of note, MedDRA has recently developed a Standardised MedDRA Query for Infective Pneumonia that can be used for analysis of pneumonia-related events [25]. Pneumonias can also be confirmed by chest radiography; these events would not necessarily be dependent on MedDRA terms, as confirmation would be performed based on investigator direction (either investigator decision or driven by protocol); however, they would probably be a subset of a larger set of pneumonia events. Finally, pneumonia events can be subject to adjudication by an independent committee, and instructions as to which events need to be adjudicated can differ between studies (supplementary figure S1).

There was great variation in the way pneumonia events were captured and recorded in studies of ICS treatment in patients with COPD (table 1). Of the trials identified, 20 evaluated the incidence of investigator-reported pneumonia with no pre-defined, adjudicated or structured approach to diagnosis (figure 2). Radiographic confirmation of suspected pneumonia cases was required for a pneumonia report in 14 trials, a standardised list of clinical signs or symptoms was used in four trials, treatment with antibiotics was required in three trials and confirmation of all pneumonia events by an independent clinical end-point committee was required in four trials (figure 2).

\section{Investigator-reported pneumonia}

In total, $22(55.0 \%)$ studies relied on investigator assessment of adverse events to report pneumonia without requiring radiographic confirmation, the presence of standardised clinical signs or symptoms, treatment with antibiotics or confirmation by an independent clinical end-point committee (table 1).

A pre-specified definition of pneumonia was not included in the protocol of the 3-year TORCH study, as the increased risk of pneumonia in patients treated with fluticasone propionate (FP)/salmeterol (SAL) or FP versus SAL or placebo was unexpected at that time [58]. Investigator-reported on-treatment pneumonia was grouped as a sponsor-defined AESI comprising 15 pneumonia-related MedDRA preferred terms [58, 59]. Investigators were not required to provide supporting evidence, such as a chest radiograph or further 
TABLE 1 Pneumonia incidence in identified COPD clinical trials, ordered by treatment comparison and pneumonia capture

$\begin{array}{llll}\text { Citation } & \text { Study and study } & \text { Study population } & \text { Pneumonia capture method }\end{array}$ length

Pneumonia incidence, \% $(\mathrm{n} / \mathrm{N})^{4}$

Increased incidence of

pneumonia versus

comparator arm

ICS arm

Comparator arm

Comparison

Fold

increase
in risk

Studies comparing ICS/LAMA/LABA triple therapy with non-ICS-containing treatment

Pneumonia capture: investigator reporting confirmed by radiographic imaging and independent adjudication

RABE, 2020 [14] ETHOS (52 weeks) Post-bronchodilator FEV F $_{1} 25-50 \%$ pred and $\geqslant 1$ moderate/severe

exacerbation or post-bronchodilator

$\mathrm{FEV}_{1} 50-65 \%$ pred and $\geqslant 2$ moderate

Investigator reported, adjudicated by

a clinical end-point independent committee

or $\geqslant 1$ severe exacerbation in the year

prior to screening

CAT total score $\geqslant 10$

Radiographic imaging compatible with

the diagnosis of pneumonia, $\geqslant 2$ of a

list of clinical signs, symptoms or

laboratory findings, and treatment

with antibiotics and/or antiviral and/

or antifungal agents were also

required to support the adjudication

FERguson, 2018 KRONOS (24 weeks) Post-bronchodilator $\mathrm{FEV}_{1} \geqslant 25 \%$ and

[26] $<80 \%$ pred

Investigator reported, adjudicated by an independent committee

Radiographic imaging compatible with daily via MDI: $1.9 \%$

the diagnosis of pneumonia, $\geqslant 2$ of a

list of clinical signs, symptoms or

laboratory findings, and treatment

with antibiotics and/or antiviral and/

or antifungal agents were also required

BUD/GLY/FOR

daily: $4.2 \%(90 /$

9.6 Hg twi

320/18/9.6 $\mu \mathrm{g}$

2144)

daily: $2.3 \%$ (48/ versus GLY/FOR

BUD/GLY/FOR 160/

18/9.6 $\mu \mathrm{g}$ twice

daily: $3.5 \%(75 /$

2124)

2125)

BUD/GLY/FOR

160/18/9.6 $\mu \mathrm{g}$

versus GLY/FOR

BUD/FOR

BUD/FOR 320/

$9.6 \mu \mathrm{g}$ twice daily:

$4.5 \%(96 / 2136)$

CAT total score $\geqslant 10$

$6 \mu \mathrm{g}$ twice daily versus GLY/FOR

via MDI: $1.6 \% \quad$ BUD/FOR 320/

BUD/FOR 320/

$(10 / 625)$

9.6 ug via MDI

$9.6 \mu \mathrm{g}$ twice daily

versus GLY/FOR

BUD/FOR 400/

$12 \mu \mathrm{g}$ via DPI

$(6 / 314)$

BUD/FOR 400/

$12 \mu \mathrm{g}$ twice daily

via DPI: $1.3 \%$ (4/

318)

Pneumonia capture: investigator reporting confirmed by radiographic imaging

ChAPMAN, 2018 SUNSET ( 26 weeks) Post-bronchodilator $\mathrm{FEV}_{1} \geqslant 40 \%$ and

[27]

$$
<80 \% \text { pred }
$$

Radiographic imaging was required to

$\leqslant 1$ moderate/severe exacerbation in confirm the diagnosis of pneumonia

the year prior to screening

LIPSON, $2018 \quad$ IMPACT (52 weeks) Post-bronchodilator $\mathrm{FEV}_{1}<50 \%$ pred

with $\geqslant 1$ moderate/severe

exacerbation in the year prior to

screening, or post-bronchodilator

$\mathrm{FEV}_{1} 50-80 \%$ pred and $\geqslant 2$ moderate

or $\geqslant 1$ severe exacerbation in the year

prior to screening

CAT total score $\geqslant 10$
Investigator reported

TIO $18 \mu \mathrm{g}$ once

daily plus SAL/FP

$50 / 500 \mu g$ twice

daily: $1.7 \%(9 / 526)$

FF/UMEC/VI 100/

$62.5 / 25 \mu \mathrm{g}$ once

Radiographic imaging was required to

confirm the diagnosis of pneumonia

daily: $7.6 \%$ (317/

4151

FF/VI 100/25 $\mu \mathrm{g}$

once daily: $7.1 \%$

(292/4134)
IND/GLY 110/ TIO+SAL/FP

$50 \mu \mathrm{g}$ once daily: versus IND/GLY $1.1 \%(6 / 527)$

UMEC/VI 62.5/ FF/UMEC/VI

$25 \mu \mathrm{g}$ once daily: versus UMEC/VI

$4.7 \%(97 / 2070) \quad \mathrm{FF} / \mathrm{VI}$ versus

UMEC/VI

\section{6}




\begin{tabular}{|c|c|c|c|c|c|c|c|}
\hline \multirow[t]{2}{*}{ Citation } & \multirow[t]{2}{*}{$\begin{array}{l}\text { Study and study } \\
\text { length }\end{array}$} & \multirow[t]{2}{*}{ Study population } & \multirow[t]{2}{*}{ Pneumonia capture method ${ }^{\#}$} & \multicolumn{2}{|c|}{ Pneumonia incidence, $\%(n / N)^{q}$} & \multicolumn{2}{|c|}{$\begin{array}{l}\text { Increased incidence of } \\
\text { pneumonia versus } \\
\text { comparator arm }\end{array}$} \\
\hline & & & & ICS arm & Comparator arm & Comparison & $\begin{array}{l}\text { Fold } \\
\text { increase } \\
\text { in risk }\end{array}$ \\
\hline $\begin{array}{l}\text { MAGNUSSEN, } \\
2014 \text { [28] }\end{array}$ & WISDOM (52 weeks) & $\begin{array}{l}\text { Post-bronchodilator } \mathrm{FEV}_{1}<50 \% \text { pred } \\
\geqslant 1 \text { exacerbation in the year prior to } \\
\text { screening }\end{array}$ & $\begin{array}{l}\text { Investigator reported } \\
\text { Radiographic imaging was requested } \\
\text { when pneumonia was suspected }\end{array}$ & $\begin{array}{c}\mathrm{FP} / \mathrm{SAL} / \mathrm{TIO} \\
(500 \mu \mathrm{g} \text { twice } \\
\text { daily/50 } \mu \mathrm{g} \text { twice } \\
\text { daily/18 } \mu \mathrm{g} \text { once } \\
\text { daily): } 5.8 \%(72 / \\
1243)\end{array}$ & $\begin{array}{c}\mathrm{SAL} / \mathrm{TIO}(50 \mu \mathrm{g} \\
\text { twice daily/18 } \mu \mathrm{g} \\
\text { once daily): } 5.5 \% \\
(68 / 1242)\end{array}$ & $\begin{array}{c}\mathrm{FP} / \mathrm{SAL} / \mathrm{TIO} \\
\text { versus } \mathrm{SAL} / \mathrm{TIO}\end{array}$ & 1.1 \\
\hline \multicolumn{8}{|c|}{ Pneumonia capture: investigator reporting } \\
\hline PAPI, 2018 [12] & TRIBUTE (52 weeks) & $\begin{array}{l}\text { Post-bronchodilator } \mathrm{FEV}_{1}<50 \% \text { pred } \\
\geqslant 1 \text { moderate/severe exacerbation in } \\
\text { the year prior to screening } \\
\text { CAT total score } \geqslant 10\end{array}$ & Investigator reported & $\begin{array}{l}\text { BDP/FOR/GLY } 87 / \\
5 / 9 \mu \mathrm{g} \text { twice daily: } \\
3.7 \%(28 / 764)\end{array}$ & $\begin{array}{c}\text { IND/GLY } 85 / \\
43 \mu \mathrm{g}: 3.6 \%(27 / \\
768)\end{array}$ & $\begin{array}{c}\mathrm{BDP} / \mathrm{FOR} / \mathrm{GLY} \\
\text { versus IND/GLY }\end{array}$ & 1.0 \\
\hline $\begin{array}{l}\text { VESTBO, } 2017 \\
{[29]}\end{array}$ & TRINITY (52 weeks) & $\begin{array}{l}\text { Post-bronchodilator } \mathrm{FEV}_{1}<50 \% \text { pred } \\
\geqslant 1 \text { moderate/severe exacerbation in } \\
\text { the year prior to screening } \\
\text { CAT total score } \geqslant 10\end{array}$ & Investigator reported & $\begin{array}{c}\text { BDP/FOR/GLY 100/ } \\
6 / 12.5 \mu \mathrm{g}(2 \\
\text { actuations twice } \\
\text { daily): } 2.6 \%(28 / \\
1077) \\
\text { BDP/FOR } 100 / 6 \mu \mathrm{g} \\
\text { (2 actuations twice } \\
\text { daily)+TIO } 18 \mu \mathrm{g}(1 \\
\text { actuation once } \\
\text { daily): } 2.2 \% \text { (12/ } \\
537)\end{array}$ & $\begin{array}{l}\text { TIO } 18 \mu \text { g once } \\
\text { daily: } 1.8 \%(19 / \\
1076)\end{array}$ & $\begin{array}{l}\mathrm{BDP} / \mathrm{FOR} / \mathrm{GLY} \\
\text { versus TIO } \\
\text { BDP/FOR+TIO } \\
\text { versus TIO }\end{array}$ & $\begin{array}{l}1.5 \\
1.3\end{array}$ \\
\hline JUNG, 2012 [30] & (24 weeks) & Post-bronchodilator $\mathrm{FEV}_{1}<65 \%$ pred & Investigator reported & $\begin{array}{l}\text { TIO } 18 \mu \text { g once } \\
\text { daily+FP/SAL } 250 / \\
50 \mu g \text { twice daily: } \\
0.9 \%(2 / 223)\end{array}$ & $\begin{array}{l}\text { TIO } 18 \mu \mathrm{g} \text { once } \\
\text { daily: } 0.9 \%(2 / \\
232)\end{array}$ & $\begin{array}{l}\text { TIO+FP/SAL } \\
\text { versus TIO }\end{array}$ & 1.0 \\
\hline \multicolumn{8}{|c|}{ Studies comparing ICS/LABA dual therapy with non-ICS-containing treatment } \\
\hline \multicolumn{8}{|c|}{ Pneumonia capture: investigator reporting and independent adjudication with/without confirmation by radiographic imaging } \\
\hline $\begin{array}{l}\text { HANANIA, } 2020 \\
{[31]}\end{array}$ & SOPHOS (52 weeks) & $\begin{array}{c}\text { Post-bronchodilator } \mathrm{FEV}_{1} \geqslant 25 \% \text { and } \\
<80 \% \text { pred } \\
\geqslant 1 \text { moderate/severe exacerbation in } \\
\text { the year prior to screening } \\
\text { CAT total score } \geqslant 10\end{array}$ & $\begin{array}{l}\text { Investigator reported, adjudicated by } \\
\text { an independent committee }\end{array}$ & $\begin{array}{l}\text { BUD/FOR 320/ } \\
10 \mu \mathrm{g} \text { twice daily: } \\
1.6 \%(10 / 619) \\
\text { BUD/FOR } 160 / \\
10 \mu \mathrm{g} \text { twice daily: } \\
2.4 \%(15 / 617)\end{array}$ & $\begin{array}{l}\text { FOR } 10 \mu g \text { twice } \\
\text { daily: } 2.3 \%(14 / \\
607)\end{array}$ & $\begin{array}{l}\text { BUD/FOR } 320 / \\
10 \mu \mathrm{g} \text { versus } \\
\text { FOR } \\
\text { BUD/FOR } 160 / \\
10 \mu \mathrm{g} \text { versus } \\
\text { FOR }\end{array}$ & $\begin{array}{l}0.7 \\
1.1\end{array}$ \\
\hline
\end{tabular}


TABLE 1 Continued

\begin{tabular}{|c|c|c|c|c|c|c|c|}
\hline \multirow[t]{2}{*}{ Citation } & \multirow[t]{2}{*}{$\begin{array}{l}\text { Study and study } \\
\text { length }\end{array}$} & \multirow[t]{2}{*}{ Study population } & \multirow[t]{2}{*}{ Pneumonia capture method ${ }^{\#}$} & \multicolumn{2}{|c|}{ Pneumonia incidence, \% $(n / N)^{4}$} & \multicolumn{2}{|c|}{$\begin{array}{l}\text { Increased incidence of } \\
\text { pneumonia versus } \\
\text { comparator arm }\end{array}$} \\
\hline & & & & ICS arm & Comparator arm & Comparison & $\begin{array}{l}\text { Fold } \\
\text { increase } \\
\text { in risk }^{+}\end{array}$ \\
\hline $\begin{array}{l}\text { FERGUSON, } 2018 \\
\quad[32]\end{array}$ & TELOS (24 weeks) & $\begin{array}{c}\text { Post-bronchodilator } \mathrm{FEV}_{1}<80 \% \text { pred } \\
\text { CAT total score } \geqslant 10\end{array}$ & $\begin{array}{c}\text { Investigator reported, adjudicated by } \\
\text { an independent committee } \\
\text { Radiographic imaging compatible with } \\
\text { the diagnosis of pneumonia, } \geqslant 2 \text { of a } \\
\text { list of clinical signs, symptoms or } \\
\text { laboratory findings, and treatment } \\
\text { with antibiotics and/or antiviral and/ } \\
\text { or antifungal agents were also } \\
\text { required }\end{array}$ & $\begin{array}{c}\text { BUD/FOR 320/ } \\
10 \mu \mathrm{g} \text { twice daily } \\
\text { via MDI: } 0.8 \% \text { (5/ } \\
655) \\
\text { BUD/FOR 160/ } \\
10 \mu \mathrm{g} \text { twice daily } \\
\text { via MDI: } 1.1 \% \text { (7/ } \\
637) \\
\text { BUD } 320 \mu \mathrm{g} \text { twice } \\
\text { daily via MDI: } 0.5 \% \\
\text { (1/206) } \\
\text { BUD/FOR 400/ } \\
12 \mu \mathrm{g} \text { twice daily } \\
\text { via DPI: } 1.4 \%(3 / \\
219)\end{array}$ & $\begin{array}{l}\text { FOR } 10 \mu \mathrm{g} \text { twice } \\
\text { daily via MDI: } \\
1.4 \%(9 / 644)\end{array}$ & $\begin{array}{c}\text { BUD/FOR 320/ } \\
10 \mu \mathrm{g} \text { versus } \\
\text { FOR } \\
\text { BUD/FOR 160/ } \\
10 \mu \mathrm{g} \text { versus } \\
\text { FOR } \\
\text { BUD versus } \\
\text { FOR } \\
\text { BUD/FOR 400/ } \\
12 \mu \mathrm{g} \text { via DPI } \\
\text { versus FOR }\end{array}$ & $\begin{array}{l}0.3 \\
1.0\end{array}$ \\
\hline \multicolumn{8}{|c|}{ Pneumonia capture: investigator reporting confirmed by radiographic imaging } \\
\hline $\begin{array}{l}\text { FERguson, } 2017 \\
\quad[33]\end{array}$ & RISE (26 weeks) & $\begin{array}{l}\text { Post-bronchodilator } \mathrm{FEV}_{1} \leqslant 70 \% \text { pred } \\
\geqslant 1 \text { moderate/severe exacerbation in } \\
\text { the year prior to screening } \\
\text { mMRC dyspnoea score } \geqslant 2\end{array}$ & $\begin{array}{l}\text { Investigator reported } \\
\text { Radiographic imaging compatible with } \\
\text { the diagnosis of pneumonia and } \geqslant 2 \\
\text { of a list of clinical signs, symptoms or } \\
\text { laboratory findings were also required }\end{array}$ & $\begin{array}{l}\text { BUD/FOR } 320 / 9 \mu \mathrm{g} \\
\text { twice daily: } 0.5 \% \\
(3 / 605)\end{array}$ & $\begin{array}{l}\text { FOR DPI } 9 \mu \mathrm{g} \\
\text { twice daily: } 1.0 \% \\
(6 / 613)\end{array}$ & $\begin{array}{l}\text { BUD/FOR } \\
\text { versus FOR }\end{array}$ & 0.5 \\
\hline PAPI, 2017 [24] & EFFECT (52 weeks) & $\begin{array}{l}\text { Post-bronchodilator } \mathrm{FEV}_{1} \leqslant 50 \% \text { pred } \\
\geqslant 1 \text { moderate/severe exacerbation in } \\
\text { the year prior to screening }\end{array}$ & $\begin{array}{c}\text { Investigator reported } \\
\text { Radiologically and/or clinically } \\
\text { confirmed per British Thoracic Society } \\
\text { criteria }\end{array}$ & $\begin{array}{l}\text { FP/FOR 500/20 } \mu \mathrm{g} \\
\text { twice daily: } 2.9 \% \\
\quad(17 / 587) \\
\text { FP/FOR } 250 / 10 \mu \mathrm{g} \\
\text { twice daily: } 3.9 \% \\
\text { (23/588) }\end{array}$ & $\begin{array}{l}\text { FOR } 12 \mu \mathrm{g} \text { twice } \\
\text { daily: } 1.9 \%(11 / \\
590)\end{array}$ & $\begin{array}{l}\text { FP/FOR 500/ } \\
20 \mu \mathrm{g} \text { versus } \\
\text { FOR } \\
\text { FP/FOR } 250 / \\
10 \mu \mathrm{g} \text { versus } \\
\text { FOR }\end{array}$ & 2.1 \\
\hline $\begin{array}{l}\text { WEDZICHA, } 2016 \\
\text { [34] }\end{array}$ & FLAME (52 weeks) & $\begin{array}{l}\text { Post-bronchodilator } \mathrm{FEV}_{1} \geqslant 25 \text { to } \\
\quad<60 \% \text { pred } \\
\geqslant 1 \text { moderate/severe exacerbation in } \\
\text { the year prior to screening } \\
\text { mMRC dyspnoea score } \geqslant 2\end{array}$ & $\begin{array}{l}\text { Investigator reported } \\
\text { Radiographic imaging was required }\end{array}$ & $\begin{array}{l}\text { FP/SAL 500/50 } \mu \mathrm{g} \\
\text { twice daily: } 4.8 \% \\
\quad(80 / 1680)\end{array}$ & $\begin{array}{l}\text { IND/GLY 110/ } \\
50 \mu \mathrm{g} \text { once daily: } \\
3.2 \% \text { (53/1678) }\end{array}$ & $\begin{array}{l}\mathrm{FP} / \mathrm{SAL} \text { versus } \\
\text { IND/GLY }\end{array}$ & 1.5 \\
\hline OHAR, 2014 [35] & $\begin{array}{l}\text { NCT01110200 } \\
\text { (26 weeks) }\end{array}$ & $\begin{array}{l}\text { Post-bronchodilator } \mathrm{FEV}_{1}<70 \% \text { pred } \\
\text { Recent exacerbation }(\leqslant 14 \text { days })\end{array}$ & $\begin{array}{l}\text { Investigator reported } \\
\text { Radiographic imaging was required }\end{array}$ & $\begin{array}{l}\mathrm{FP} / \mathrm{SAL} 250 / 50 \mu \mathrm{g} \\
\text { twice daily: } 4.1 \% \\
\quad(13 / 314)\end{array}$ & $\begin{array}{l}\text { SAL } 50 \mu \text { twice } \\
\text { daily: } 3.1 \%(10 / \\
325)\end{array}$ & $\begin{array}{l}\mathrm{FP} / \mathrm{SAL} \text { versus } \\
\text { SAL }\end{array}$ & 1.3 \\
\hline
\end{tabular}




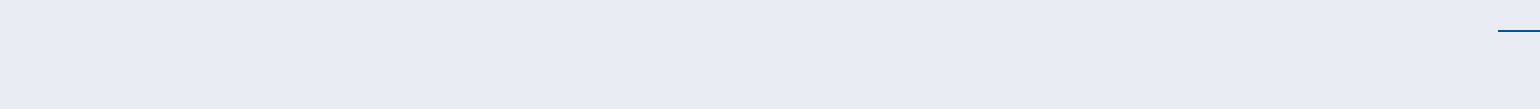

\begin{tabular}{|c|c|c|c|c|c|c|c|}
\hline $\begin{array}{l}\text { VogeLmeIeR, } \\
2013[36]\end{array}$ & ILLUMINATE (26 weeks) & $\begin{array}{c}\text { Post-bronchodilator } \mathrm{FEV}_{1} 40-80 \% \\
\text { pred } \\
0 \text { moderate/severe exacerbations in } \\
\text { the year prior to screening }\end{array}$ & $\begin{array}{l}\text { Investigator reported } \\
\text { Radiographic imaging was required }\end{array}$ & $\begin{array}{l}\text { FP/SAL } 500 / 50 \mu \mathrm{g} \\
\text { twice daily: } 1.5 \% \\
\quad(4 / 264)\end{array}$ & $\begin{array}{c}\text { IND/GLY } 110 / \\
50 \mu \mathrm{g} \text { once daily: } \\
0(0 / 258)\end{array}$ & & \\
\hline $\begin{array}{l}\text { Anzueto, } 2009 \\
{[10]}\end{array}$ & $\begin{array}{l}\text { NCT00115492 } \\
\text { (52 weeks) }\end{array}$ & $\begin{array}{c}\text { Post-bronchodilator } \mathrm{FEV}_{1} \leqslant 50 \% \text { pred } \\
\geqslant 1 \text { moderate/severe exacerbation in } \\
\text { the year prior to screening }\end{array}$ & $\begin{array}{l}\text { Investigator reported } \\
\text { Radiographic imaging was required }\end{array}$ & $\begin{array}{l}\text { FP/SAL 250/50 } \mu \mathrm{g} \\
\text { twice daily: } 6.6 \% \\
(26 / 394)\end{array}$ & $\begin{array}{l}\text { SAL } 50 \mu \mathrm{g} \text { twice } \\
\text { daily: } 2.5 \%(10 / \\
403)\end{array}$ & $\begin{array}{l}\mathrm{FP} / \mathrm{SAL} \text { versus } \\
\text { SAL }\end{array}$ & 2.7 \\
\hline \multicolumn{8}{|c|}{ Pneumonia capture: investigator reporting } \\
\hline $\begin{array}{l}\text { SUISSA, } 2018 \\
{[37]}\end{array}$ & Up to 1 -year follow-up & $\begin{array}{l}\text { Cohort of patients aged } \geqslant 55 \text { years } \\
\text { with COPD initiating treatment with a } \\
\text { LAMA or ICS/LABA during 2002-2015 } \\
\text { from the UK's Clinical Practice } \\
\text { Research Datalink }\end{array}$ & $\begin{array}{l}\text { Hospital admissions due to severe } \\
\text { pneumonia (according to diagnostic } \\
\text { codes) }\end{array}$ & $\begin{array}{l}\text { ICS/LABA: } 3.1 \% \\
(380 / 12366)\end{array}$ & $\begin{array}{l}\text { LAMA: } 2.3 \%(279 / \\
12366) \text { of } \\
\text { patients }\end{array}$ & $\begin{array}{l}\text { ICS/LABA } \\
\text { versus LAMA }\end{array}$ & 1.4 \\
\hline $\begin{array}{l}\text { VESTBO, } 2016 \\
\quad[38] \\
\text { CRIM, } 2017 \\
\text { [39] }\end{array}$ & $\begin{array}{l}\text { SUMMIT (event driven, } \\
\text { common end date of } \\
3 \text { years) }\end{array}$ & $\begin{array}{c}\text { Post-bronchodilator } \mathrm{FEV}_{1} \geqslant 50 \% \text { and } \\
\leqslant 70 \% \text { pred } \\
\text { History or increased risk of } \\
\text { cardiovascular disease } \\
\text { mMRC dyspnoea score } \geqslant 2\end{array}$ & Investigator reported & $\begin{array}{l}\text { FF/VI } 100 / 25 \mu \mathrm{g} \\
\text { once daily: } 5.7 \% \\
\quad(237 / 4140) \\
\text { FF } 100 \mu \mathrm{g} \text { once } \\
\text { daily: } 5.5 \%(228 / \\
4157)\end{array}$ & $\begin{array}{c}\text { VI } 25 \mu \text { g once } \\
\text { daily: } 3.9 \%(163 / \\
4140) \\
\text { Placebo: } 5.2 \% \\
\text { (214/4131) }\end{array}$ & $\begin{array}{l}\mathrm{FF} / \mathrm{VI} \text { versus } \mathrm{VI} \\
\mathrm{FF} \text { versus } \\
\text { placebo }\end{array}$ & $\begin{array}{l}1.5 \\
1.1\end{array}$ \\
\hline $\begin{array}{l}\text { VestBo, } 2016 \\
{[40]}\end{array}$ & $\begin{array}{l}\text { Salford Lung Study } \\
\text { (52 weeks) }\end{array}$ & $\begin{array}{l}\geqslant 1 \text { COPD exacerbations in the } \\
\text { previous } 3 \text { years }\end{array}$ & Investigator reported pneumonia SAEs & $\begin{array}{c}\text { FF/VI } 100 / 25 \mu \mathrm{g} \\
\text { once daily: } 6.7 \% \\
\text { (94/1396) }\end{array}$ & $\begin{array}{l}\text { Usual care: } 5.9 \% \\
\quad(83 / 1403)\end{array}$ & $\begin{array}{l}\mathrm{FF} / \mathrm{VI} 100 / \\
25 \mu \mathrm{g} \text { versus } \\
\text { usual care }\end{array}$ & 1.1 \\
\hline $\begin{array}{l}\text { VogeLMEIER, } \\
2016[41]\end{array}$ & AFFIRM (24 weeks) & $\begin{array}{l}\text { Post-bronchodilator } \mathrm{FEV}_{1}<80 \% \text { pred } \\
\text { CAT total score } \geqslant 10\end{array}$ & Investigator reported & $\begin{array}{l}\text { FP/SAL 500/50 } \mu \mathrm{g} \\
\text { twice daily: } 1.9 \% \\
(9 / 466)\end{array}$ & $\begin{array}{l}\text { ACL/FOR 400/ } \\
12 \mu \mathrm{g} \text { twice daily: } \\
0.6 \%(3 / 467)\end{array}$ & $\begin{array}{l}\text { FP/SAL versus } \\
\text { ACL/FOR }\end{array}$ & 3.2 \\
\hline $\begin{array}{l}\text { ZHENG, } 2015 \\
\quad[42]\end{array}$ & $\begin{array}{l}\text { NCT01376245 } \\
\text { (24 weeks) }\end{array}$ & $\begin{array}{c}\text { Asian patients } \\
\text { Post-bronchodilator } \mathrm{FEV}_{1} \leqslant 70 \% \text { pred } \\
\text { mMRC dyspnoea score } \geqslant 2\end{array}$ & Investigator reported & 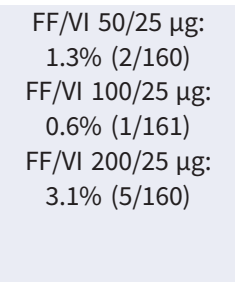 & $\begin{array}{c}\text { Placebo: } 2.5 \%(4 / \\
162)\end{array}$ & $\begin{array}{c}\text { FF/VI } 50 / 25 \mu \mathrm{g} \\
\text { versus placebo } \\
\text { FF/VI 100/ } \\
25 \mu \mathrm{g} \text { versus } \\
\text { placebo } \\
\text { FF/VI 200/ } \\
25 \mu \mathrm{g} \text { versus } \\
\text { placebo }\end{array}$ & $\begin{array}{l}0.5 \\
0.3\end{array}$ \\
\hline $\begin{array}{l}\text { ZHONG, } 2015 \\
\quad[43]\end{array}$ & LANTERN (26 weeks) & $\begin{array}{c}\text { Post-bronchodilator } \mathrm{FEV}_{1} \geqslant 30 \% \text { and } \\
<80 \% \text { pred } \\
\leqslant 1 \text { moderate/severe exacerbation in } \\
\text { the year prior to screening } \\
\text { mMRC dyspnoea score } \geqslant 2\end{array}$ & Investigator reported & $\begin{array}{l}\text { FP/SAL 500/50 } \mu \mathrm{g}: \\
2.7 \%(10 / 369)\end{array}$ & $\begin{array}{l}\text { IND/GLY } 110 / \\
50 \mu g: 0.8 \%(3 / \\
372)\end{array}$ & $\begin{array}{l}\text { FP/SAL versus } \\
\text { IND/GLY }\end{array}$ & 3.4 \\
\hline
\end{tabular}




\begin{tabular}{|c|c|c|c|c|c|c|c|}
\hline \multirow[t]{2}{*}{ Citation } & \multirow[t]{2}{*}{$\begin{array}{l}\text { Study and study } \\
\text { length }\end{array}$} & \multirow[t]{2}{*}{ Study population } & \multirow[t]{2}{*}{ Pneumonia capture method ${ }^{\#}$} & \multicolumn{2}{|c|}{ Pneumonia incidence, $\%(n / N)^{\natural}$} & \multicolumn{2}{|c|}{$\begin{array}{l}\text { Increased incidence of } \\
\text { pneumonia versus } \\
\text { comparator arm }\end{array}$} \\
\hline & & & & ICS arm & Comparator arm & Comparison & $\begin{array}{l}\text { Fold } \\
\text { increase } \\
\text { in risk }^{+}\end{array}$ \\
\hline $\begin{array}{l}\text { Rossl, } 2014 \\
\quad[44]\end{array}$ & INSTEAD (26 weeks) & $\begin{array}{l}\text { GOLD stage II } \\
0 \text { moderate/severe exacerbation in } \\
\text { the year prior to screening }\end{array}$ & Investigator-reported pneumonia SAEs & $\begin{array}{l}\text { FP/SAL } 500 / 50 \mu \mathrm{g} \\
\text { twice daily: } 0.7 \% \\
\text { (2/288) }\end{array}$ & $\begin{array}{l}\text { IND } 150 \mu \mathrm{g} \text { once } \\
\text { daily: } 0(0 / 293)\end{array}$ & $\begin{array}{l}\text { FP/SAL versus } \\
\text { IND }\end{array}$ & \\
\hline $\begin{array}{l}\text { WEDZICHA, } 2014 \\
{[45]}\end{array}$ & FORWARD (48 weeks) & $\begin{array}{l}\text { Post-bronchodilator } \mathrm{FEV}_{1} \geqslant 30 \% \text { and } \\
\qquad<50 \% \text { pred } \\
\geqslant 1 \text { moderate/severe exacerbation in } \\
\text { the year prior to screening }\end{array}$ & Investigator reported & $\begin{array}{l}\text { BDP/FOR 200/ } \\
12 \mu \mathrm{g} \text { twice daily: } \\
3.8 \%(23 / 601)\end{array}$ & $\begin{array}{l}\text { FOR } 12 \mu \mathrm{g} \text { twice } \\
\text { daily: } 1.8 \%(11 / \\
596)\end{array}$ & $\begin{array}{l}\text { BDP/FOR } \\
\text { versus FOR }\end{array}$ & 2.1 \\
\hline $\begin{array}{l}\text { DRANSField, } \\
2013 \text { [46], } \\
\text { CRIM, 2015 } \\
\text { [47] }\end{array}$ & $\begin{array}{l}\text { NCT01009463, } \\
\text { NCT01017952 } \\
\text { (52 weeks) }\end{array}$ & $\begin{array}{l}\text { Post-bronchodilator } \mathrm{FEV}_{1}<70 \% \text { pred } \\
\geqslant 1 \text { moderate/severe exacerbation in } \\
\text { the year prior to screening }\end{array}$ & Investigator reported & $\begin{array}{c}\mathrm{FF} / \mathrm{VI} 200 / 25 \mu \mathrm{g} \\
\text { once daily: } 6.8 \% \\
\text { (55/811) } \\
\text { FF/VI 100/25 } \mu \mathrm{g} \\
\text { once daily: } 6.3 \% \\
\text { (51/806) } \\
\text { FF/VI 50/25 } \mu \mathrm{g} \\
\text { once daily: } 5.9 \% \\
\text { (48/820) }\end{array}$ & $\begin{array}{c}\text { VI } 25 \mu \text { g once } \\
\text { daily: } 3.3 \%(27 / \\
818)\end{array}$ & $\begin{array}{c}\mathrm{FF} / \mathrm{VI} 50 / 25 \mu \mathrm{g} \\
\text { versus } \mathrm{VI} \\
\mathrm{FF} / \mathrm{VI} 100 / \\
25 \mu \mathrm{g} \text { versus VI } \\
\mathrm{FF} / \mathrm{VI} 200 / \\
25 \mu \mathrm{g} \text { versus VI }\end{array}$ & $\begin{array}{l}1.8 \\
1.9 \\
2.1\end{array}$ \\
\hline $\begin{array}{l}\text { KERWIN, } 2013 \\
\text { [48] }\end{array}$ & $\begin{array}{l}\text { NCT01053988 } \\
\text { (24 weeks) }\end{array}$ & $\begin{array}{c}\text { Post-bronchodilator } \mathrm{FEV}_{1} \leqslant 70 \% \text { pred } \\
\text { mMRC dyspnoea score } \geqslant 2\end{array}$ & Investigator reported & $\begin{array}{c}\text { FF/VI } 100 / 25 \mu \mathrm{g} \\
\text { once daily: } 2.4 \% \\
(5 / 206) \\
\text { FF/VI } 50 / 25 \mu \mathrm{g} \\
\text { once daily: } 1.5 \% \\
(3 / 206) \\
\text { FF } 100 \mu \mathrm{g} \text { once } \\
\text { daily: } 1.9 \%(4 / 206)\end{array}$ & $\begin{array}{c}\text { VI } 25 \mu \mathrm{g} \text { once } \\
\text { daily: } 2.4 \%(5 / \\
205) \\
\text { Placebo: } 1.4 \% \text { (3/ } \\
207)\end{array}$ & $\begin{array}{c}\mathrm{FF} / \mathrm{VI} 100 / \\
25 \mu \mathrm{g} \text { versus } \mathrm{VI} \\
\mathrm{FF} / \mathrm{VI} 50 / 25 \mu \mathrm{g} \\
\text { versus } \mathrm{VI} \\
\mathrm{FF} \text { versus } \\
\text { placebo }\end{array}$ & $\begin{array}{l}1.0 \\
0.6 \\
1.3\end{array}$ \\
\hline $\begin{array}{l}\text { MaRTINEZ, } 2013 \\
\quad[49]\end{array}$ & $\begin{array}{l}\text { NCT01054885 } \\
\text { (24 weeks) }\end{array}$ & $\begin{array}{c}\text { Post-bronchodilator } \mathrm{FEV}_{1} \leqslant 70 \% \text { pred } \\
\text { mMRC dyspnoea score } \geqslant 2\end{array}$ & Investigator reported & $\begin{array}{c}\text { FF/VI } 200 / 25 \mu \mathrm{g} \\
\text { once daily: } 2.0 \% \\
(4 / 205) \\
\text { FF/VI } 100 / 25 \mu \mathrm{g} \\
\text { once daily: } 0.5 \% \\
(1 / 204) \\
\text { FF } 200 \mu \mathrm{g} \text { once } \\
\text { daily: } 1.5 \%(3 / 203) \\
\text { FF } 100 \mu \mathrm{g} \text { once } \\
\text { daily: } 1.0 \%(2 / 204)\end{array}$ & $\begin{array}{l}\text { VI } 25 \mu \text { g once } \\
\text { daily: } 1.0 \%(2 / \\
203) \\
\text { Placebo: } 0(0 / \\
205)\end{array}$ & $\begin{array}{c}\mathrm{FF} / \mathrm{VI} 200 / \\
25 \mu \mathrm{g} \text { versus } \mathrm{VI} \\
\mathrm{FF} / \mathrm{VI} 100 / \\
25 \mu \mathrm{g} \text { versus VI } \\
\mathrm{FF} 200 \mu \mathrm{g} \\
\text { versus } \mathrm{VI} \\
\mathrm{FF} 100 \mu \mathrm{g} \\
\text { versus } \mathrm{VI}\end{array}$ & $\begin{array}{l}2.0 \\
0.5 \\
1.5 \\
1.0\end{array}$ \\
\hline
\end{tabular}




\begin{tabular}{|c|c|c|c|c|c|c|c|}
\hline \multirow[t]{2}{*}{ Citation } & \multirow[t]{2}{*}{$\begin{array}{l}\text { Study and study } \\
\text { length }\end{array}$} & \multirow[t]{2}{*}{ Study population } & \multirow[t]{2}{*}{ Pneumonia capture method } & \multicolumn{2}{|c|}{ Pneumonia incidence, $\%(n / N)^{थ}$} & \multicolumn{2}{|c|}{$\begin{array}{l}\text { Increased incidence of } \\
\text { pneumonia versus } \\
\text { comparator arm }\end{array}$} \\
\hline & & & & ICS arm & Comparator arm & Comparison & $\begin{array}{l}\text { Fold } \\
\text { increase } \\
\text { in risk }\end{array}$ \\
\hline $\begin{array}{l}\text { DOHERTY, } 2012 \\
\text { [50] }\end{array}$ & (52 weeks) & $\begin{array}{l}\text { Post-bronchodilator } \mathrm{FEV}_{1} 25-60 \% \\
\text { pred } \\
\text { Symptoms of COPD for } \geqslant 24 \text { months } \\
\text { prior to enrolment }\end{array}$ & Investigator reported & $\begin{array}{c}\text { MF/FOR 400/10 } \mu \mathrm{g} \\
\text { twice daily: } 3.1 \% \\
\text { (7/225) } \\
\text { MF/FOR 200/10 } \mu \mathrm{g} \\
\text { twice daily: } 1.7 \% \\
(4 / 239) \\
\text { MF } 400 \mu \mathrm{g} \text { twice } \\
\text { daily: } 2.0 \%(5 / 253)\end{array}$ & $\begin{array}{l}\text { FOR } 10 \mu \mathrm{g} \text { twice } \\
\text { daily: } 1.6 \%(4 / \\
243) \\
\text { Placebo: } 0.8 \%(2 / \\
236)\end{array}$ & $\begin{array}{c}\text { MF/FOR 400/ } \\
10 \mu g \text { versus } \\
\text { FOR } \\
\text { MF/FOR 200/ } \\
10 \mu g \text { versus } \\
\text { FOR } \\
\text { MF versus } \\
\text { placebo }\end{array}$ & 2.3 \\
\hline $\begin{array}{l}\text { TASHIIN, } 2012 \\
\text { [51] }\end{array}$ & $\begin{array}{c}\text { NCT00383435 } \\
\text { (52 weeks) }\end{array}$ & $\begin{array}{c}\text { Post-bronchodilator } \mathrm{FEV}_{1} \geqslant 25 \% \text { and } \\
\leqslant 60 \% \text { pred } \\
\text { Symptoms of COPD for } \geqslant 24 \text { months }\end{array}$ & Investigator reported & $\begin{array}{c}\text { MF/FOR 400/10 } \mu \mathrm{g} \\
\text { twice daily: } 1.8 \% \\
(4 / 217) \\
\text { MF/FOR } 200 / 10 \mu \mathrm{g} \\
\text { twice daily: } 0.5 \% \\
(1 / 207) \\
\text { MF } 400 \mu \mathrm{g} \text { twice } \\
\text { daily: } 1.0 \%(2 / 210)\end{array}$ & $\begin{array}{l}\text { FOR } 10 \mu g \text { twice } \\
\text { daily: } 1.9 \%(4 / \\
209)\end{array}$ & $\begin{array}{c}\text { MF/FOR 400/ } \\
10 \mu g \text { versus } \\
\text { FOR } \\
\text { MF/FOR 200/ } \\
10 \mu \mathrm{g} \text { versus } \\
\text { FOR } \\
\text { MF versus FOR }\end{array}$ & 0.5 \\
\hline $\begin{array}{l}\text { SHARAFKHANEH, } \\
2012 \text { [52] }\end{array}$ & $\begin{array}{l}\text { NCT00419744 } \\
\text { (52 weeks) }\end{array}$ & $\begin{array}{l}\text { Pre-bronchodilator } \mathrm{FEV}_{1} \leqslant 50 \% \text { pred } \\
\geqslant 1 \text { moderate/severe exacerbation in } \\
\text { the year prior to screening }\end{array}$ & Investigator reported & $\begin{array}{l}\text { BUD/FOR } 320 / 9 \mu \mathrm{g} \\
\text { twice daily: } 6.4 \% \\
\text { (26/407) } \\
\text { BUD/FOR } 160 / 9 \mu \mathrm{g}: \\
4.7 \%(19 / 408)\end{array}$ & $\begin{array}{l}\text { FOR } 9 \mu \text { g twice } \\
\text { daily: } 2.7 \%(11 / \\
403)\end{array}$ & $\begin{array}{l}\text { BUD/FOR } 320 / \\
9 \mu \mathrm{g} \text { versus } \\
\text { FOR } \\
\text { BUD/FOR } 160 / \\
9 \mu \mathrm{g} \text { versus } \\
\text { FOR }\end{array}$ & 1.7 \\
\hline $\begin{array}{l}\text { CalverLey, } 2010 \\
\text { [53] }\end{array}$ & $\begin{array}{l}\text { NCT00476099 } \\
\text { (48 weeks) }\end{array}$ & $\begin{array}{l}\text { Post-bronchodilator } \mathrm{FEV}_{1} 30-50 \% \\
\text { pred } \\
\geqslant 1 \text { moderate/severe exacerbation in } \\
\text { the year prior to screening }\end{array}$ & Investigator reported & $\begin{array}{c}\text { BDP/FOR 200/ } \\
12 \mu \mathrm{g} \text { twice daily: } \\
2.2 \%(5 / 232) \\
\text { BUD/FOR 400/ } \\
12 \mu \mathrm{g} \text { twice daily: } \\
2.9 \%(7 / 238)\end{array}$ & $\begin{array}{l}\text { FOR } 12 \mu \mathrm{g} \text { twice } \\
\text { daily: } 0.4 \%(1 / \\
233)\end{array}$ & $\begin{array}{c}\text { BDP/FOR } \\
\text { versus FOR } \\
\text { BUD/FOR } \\
\text { versus FOR }\end{array}$ & $\begin{array}{l}5.0 \\
6.9\end{array}$ \\
\hline $\begin{array}{l}\text { RENNARD, } 2009 \\
\text { [54] }\end{array}$ & $\begin{array}{c}\text { NCT00206167 } \\
\text { (52 weeks) }\end{array}$ & $\begin{array}{l}\text { Post-bronchodilator } \mathrm{FEV}_{1} \leqslant 50 \% \text { pred } \\
\geqslant 1 \text { moderate/severe exacerbation in } \\
\text { the year prior to screening } \\
\text { mMRC dyspnoea score } \geqslant 2\end{array}$ & Investigator reported & $\begin{array}{l}\text { BUD/FOR 320/9 } \mu \mathrm{g} \\
\text { twice daily: } 4.0 \% \\
(20 / 494) \\
\text { BUD/FOR } 160 / 9 \mu \mathrm{g} \\
\text { twice daily: } 3.4 \% \\
(17 / 494)\end{array}$ & $\begin{array}{l}\text { FOR } 9 \mu \text { twice } \\
\text { daily: } 3.4 \%(17 / \\
495) \\
\text { Placebo: } 5.0 \% \\
\text { (24/481) }\end{array}$ & $\begin{array}{l}\text { BUD/FOR } 320 / \\
9 \mu \mathrm{g} \text { versus } \\
\text { FOR } \\
\text { BUD/FOR } 160 / \\
9 \mu \mathrm{g} \text { versus } \\
\text { FOR }\end{array}$ & 1.0 \\
\hline $\begin{array}{l}\text { FERGUSON, } 2008 \\
\text { [11] }\end{array}$ & $\begin{array}{l}\text { NCT00144911 } \\
\text { (52 weeks) }\end{array}$ & $\begin{array}{c}\text { Post-bronchodilator } \mathrm{FEV}_{1} \leqslant 50 \% \text { pred } \\
\geqslant 1 \text { moderate/severe exacerbation in } \\
\text { the year prior to screening }\end{array}$ & Investigator reported & $\begin{array}{l}\text { FP/SAL } 250 / 50 \mu \mathrm{g} \\
\text { twice daily: } 7.4 \% \\
\text { (29/394) }\end{array}$ & $\begin{array}{l}\text { SAL } 50 \mu \mathrm{g} \text { twice } \\
\text { daily: } 3.9 \%(15 / \\
388)\end{array}$ & $\begin{array}{c}\mathrm{FP} / \mathrm{SAL} \text { versus } \\
\text { SAL }\end{array}$ & 1.9 \\
\hline
\end{tabular}


TABLE 1 Continued

\begin{tabular}{|c|c|c|c|c|c|c|c|}
\hline \multirow[t]{2}{*}{ Citation } & \multirow[t]{2}{*}{$\begin{array}{l}\text { Study and study } \\
\text { length }\end{array}$} & \multirow[t]{2}{*}{ Study population } & \multirow[t]{2}{*}{ Pneumonia capture method ${ }^{\#}$} & \multicolumn{2}{|c|}{ Pneumonia incidence, $\%(n / N)^{4}$} & \multicolumn{2}{|c|}{$\begin{array}{l}\text { Increased incidence of } \\
\text { pneumonia versus } \\
\text { comparator arm }\end{array}$} \\
\hline & & & & ICS arm & Comparator arm & Comparison & $\begin{array}{l}\text { Fold } \\
\text { increase } \\
\text { in risk }\end{array}$ \\
\hline $\begin{array}{l}\text { TASHKIN, } 2008 \\
{[55]}\end{array}$ & $\begin{array}{l}\text { NCT00206154 } \\
\text { (26 weeks) }\end{array}$ & $\begin{array}{l}\text { Pre-bronchodilator } \mathrm{FEV}_{1} \leqslant 50 \% \text { pred } \\
\geqslant 1 \text { moderate/severe exacerbation in } \\
\text { the year prior to screening } \\
\text { mMRC dyspnoea score } \geqslant 2\end{array}$ & Investigator reported & $\begin{array}{c}\text { BUD/FOR } 320 / 9 \mu \mathrm{g} \\
\text { twice daily: } 0.4 \% \\
\text { (1/277) } \\
\text { BUD/FOR } 160 / 9 \mu \mathrm{g} \\
\text { twice daily: } 0.7 \% \\
\text { (2/281) } \\
\text { BUD } 320 \mu \mathrm{g} \text { twice } \\
\text { daily+FOR } 9 \mu \mathrm{g} \\
\text { twice daily: } 0.7 \% \\
\text { (2/287) } \\
\text { BUD } 320 \mu \mathrm{g} \text { twice } \\
\text { daily: } 1.1 \%(3 / 275)\end{array}$ & $\begin{array}{l}\text { FOR } 9 \mu \mathrm{g} \text { twice } \\
\text { daily: } 0.4 \%(1 / \\
284) \\
\text { Placebo: } 0.3 \%(1 / \\
300)\end{array}$ & $\begin{array}{c}\text { BUD/FOR 320/ } \\
9 \mu \mathrm{g} \text { versus } \\
\text { FOR } \\
\text { BUD/FOR 160/ } \\
9 \mu \mathrm{g} \text { versus } \\
\text { FOR } \\
\text { BUD } 320 \mu \mathrm{g} \\
\text { +FOR } 9 \mu \mathrm{g} \\
\text { versus FOR } \\
\text { BUD versus } \\
\text { placebo }\end{array}$ & 2.0 \\
\hline $\begin{array}{l}\text { WEDZICHA, } 2008 \\
\text { [56], } \\
\text { CALVERLEY, } \\
2011[57]\end{array}$ & INSPIRE (2 years) & $\begin{array}{l}\text { Post-bronchodilator } \mathrm{FEV}_{1}<50 \% \text { pred, } \\
\text { mMRC dyspnoea score } \geqslant 2 \text {, clinical } \\
\text { history of exacerbations }\end{array}$ & Investigator reported & $\begin{array}{l}\text { FP/SAL } 500 / 50 \mu \mathrm{g} \\
\text { twice daily: } 7.6 \% \\
\quad(50 / 658)\end{array}$ & $\begin{array}{l}\text { TIO } 18 \mu \mathrm{g} \text { once } \\
\text { daily: } 3.6 \%(24 / \\
665)\end{array}$ & $\begin{array}{c}\text { FP/SAL versus } \\
\text { TIO }\end{array}$ & 2.1 \\
\hline $\begin{array}{l}\text { Calverley, } 2007 \\
\text { [58], } \\
\text { CRIM, } 2009 \\
\text { [59] }\end{array}$ & $\begin{array}{l}\text { TORCH (3 years) } \\
\text { (pneumonia incidences } \\
\text { from a post hoc } \\
\text { analysis focusing on } \\
\text { pneumonia are } \\
\text { reported here) }\end{array}$ & Pre-bronchodilator $\mathrm{FEV}_{1}<60 \%$ pred & Investigator reported & $\begin{array}{l}\text { FP } 500 \mu \mathrm{gg} \text { twice } \\
\text { daily: } 14.4 \%(224 / \\
1552) \\
\text { FP/SAL } 500 / 50 \mu \mathrm{g} \\
\text { twice daily: } 16.0 \% \\
\text { (248/1546) }\end{array}$ & $\begin{array}{l}\text { SAL } 50 \mu g \text { twice } \\
\text { daily: 10.5\% } \\
\text { (162/1542) } \\
\text { Placebo: } 9.0 \% \\
\text { (139/1544) }\end{array}$ & $\begin{array}{l}\text { FP/SAL versus } \\
\text { SAL } \\
\text { FP versus } \\
\text { placebo }\end{array}$ & 1.5 \\
\hline
\end{tabular}

ICS: inhaled corticosteroid; LAMA: long-acting muscarinic antagonist; LABA: long-acting $\beta_{2}$-agonist; FEV $_{1}$ : forced expiratory volume in $1 \mathrm{~s}$; CAT: COPD Assessment Test; BUD: budesonide; GLY: glycopyrronium; FOR: formoterol; MDI: metered-dose inhaler; DPI: dry powder inhaler; TIO: tiotropium; SAL: salmeterol; FP: fluticasone propionate; FF: fluticasone furoate; UMEC: umeclidinium; VI: vilanterol; BDP: beclomethasone dipropionate; mMRC: modified Medical Research Council; SAE: serious adverse event; ACL: aclidinium; IND: indacaterol; GOLD: Global Initiative for Chronic Obstructive Lung Disease; MF: mometasone furoate. ": when a study uses different sets of criteria to define pneumonia and reports different pneumonia incidences when using these different criteria, only the pneumonia incidence for the most stringent pneumonia capture method is reported. The corresponding method is summarised in the "pneumonia capture method" column; ${ }^{\top}: \mathrm{N}$ is the total number of patients in the subgroup of interest, $\mathrm{n}$ the number of patients with events; ${ }^{+}$: fold increase calculated as (incidence in treatment arm of interest)/(incidence in comparator arm). 


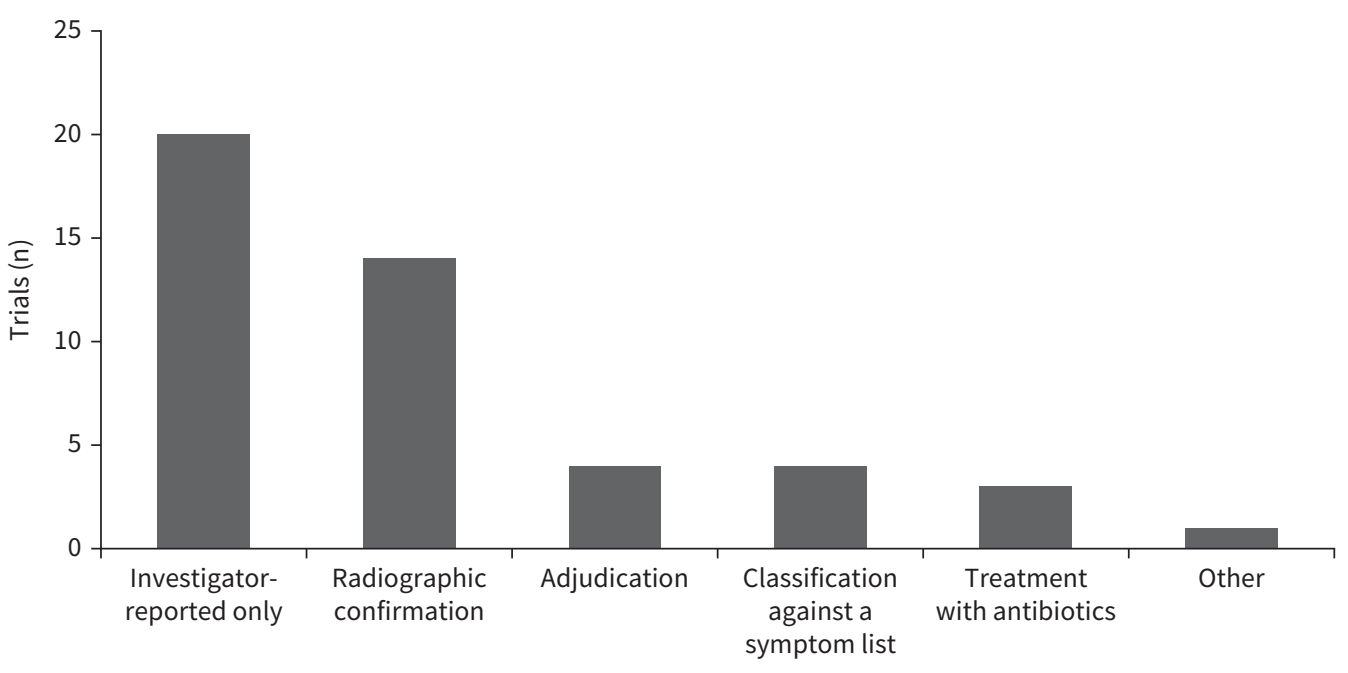

Requirements for pneumonia capture

FIGURE 2 COPD trials by pneumonia capture methodology. Some trials included multiple pneumonia capture methodologies.

laboratory tests with respect to pneumonia events [58, 59]. In a post hoc analysis of TORCH, these on-treatment pneumonia AESIs over the 3-year study period were reported for $16.0 \%(n=248), 14.4 \%$ $(n=224)$ and $10.5 \%(n=162)$ of patients treated with FP/SAL, FP and SAL, respectively, representing a 1.5-fold increased risk of pneumonia with FP/SAL versus SAL (table 1) [58, 59].

In the phase III 52-week IMPACT study, patients were randomised 2:2:1 to treatment with fluticasone furoate (FF), umeclidinium (UMEC) and vilanterol (VI), FF/VI or UMEC/VI, administered in a single inhaler [13]. Investigator-reported pneumonia was evaluated as a sponsor-defined AESI comprising 70 pneumonia-related MedDRA preferred terms. Events reported as pneumonia by the investigator required confirmation by the presence of new infiltrate(s) on a chest radiograph and at least two clinical signs from a pre-defined list. Pneumonia events were reported for 7.6\% (n=317), 7.1\% ( $n=292)$ and 4.7\% ( $=97)$ of patients treated with FF/UMEC/VI, FF/VI and UMEC/VI, respectively [13].

DrANSFIELD et al. [46] reported the findings of two replicate 1-year trials that compared treatment with VI $25 \mu \mathrm{g}$ combined with FF 50, 100 or $200 \mu \mathrm{g}$ versus treatment with VI $25 \mu \mathrm{g}$ alone. Pneumonia was assessed as a sponsor-defined AESI of 48 pneumonia-related MedDRA preferred terms [47], and was reported for $5.9 \%(n=48), 6.3 \%(n=51), 6.8 \%(n=55)$ and $3.3 \%(n=27)$ of patients assigned to these treatment groups, respectively, representing a 1.8-2.1-fold increased risk of pneumonia with FF/VI versus VI [46]. In contrast, in the 1-year TRIBUTE study, comparing beclomethasone dipropionate (BDP)/ glycopyrrolate (GLY)/formoterol (FOR) with indacaterol (IND)/GLY, pneumonia was assessed using a narrower sponsor-defined AESI of seven pneumonia-related MedDRA preferred terms. Pneumonia was reported in 3.7\% $(n=28)$ of patients treated with BDP/GLY/FOR and 3.6\% $(n=27)$ of those treated with IND/GLY; no increased risk of pneumonia was seen with this triple therapy versus the long-acting $\beta_{2}$-agonist (LABA)/long-acting muscarinic antagonist (LAMA) combination [12]. In contrast, SHARAFKHANEH et al. [52] reported pneumonia, assessed using six preferred terms, for $6.4 \%(\mathrm{n}=26)$ and 4.7\% ( $\mathrm{n}=19)$ of patients treated with BDP/FOR 320/9 $\mu \mathrm{g}$ and BDP/FOR 160/9 $\mu \mathrm{g}$, demonstrating a 2.3- and 1.7-fold increased risk of pneumonia, respectively, versus FOR $9 \mu \mathrm{g}(2.7 \%, \mathrm{n}=11)$.

It should be noted that in studies that only used investigator reporting of adverse events and AESIs to capture pneumonia, differences in the incidence of pneumonia events were also influenced by other factors, such as patient population and length of study, in addition to the number of preferred terms used. Indeed, increasing the number of preferred terms used to define pneumonia is likely to capture less frequently reported terms and therefore may not have as big an impact on the incidence reported as other factors, such as patient population and length of study. Nonetheless, while these studies varied in design and duration, they tended to report marginally higher incidences of pneumonia than those in which additional methods were used to guide reporting of pneumonias, such as the presence of standardised symptoms, radiological confirmation or adjudication by an independent clinical end-point committee. 


\section{Radiographic imaging}

In addition to investigator reporting of pneumonia adverse events, some RCTs have required supporting radiographic imaging for a report of pneumonia to be made; in this review 33.3\% (12 out of 36) trials required supporting radiographic confirmation. It is important to note that the accuracy of supportive radiographic imaging can differ depending on where and how it is conducted, the clinical scenario of each individual patient and whether the radiographic assessment is done by a single radiologist or a panel who review the images in a blinded fashion. Additionally, it is often not clear whether the report of pneumonia in a given study followed radiological reporting alone, or included physician review. In addition, radiographic imaging practices vary by country, with computed tomography most frequently used in Japan and the United States [23, 60]. Furthermore, some trial protocols, such as the one for IMPACT, asked for chest radiography to be conducted within $48 \mathrm{~h}$ of both pneumonia and exacerbation events [13,61], which may increase the identification of pneumonia that otherwise would go unrecognised. Conversely, this additional diagnostic criterion of chest radiography may contribute to a lower reported incidence of pneumonia compared with relying on investigator reports of pneumonia adverse events alone. For example, while the 1-year EFFECT study reported radiologically and/or clinically defined pneumonia per British Thoracic Society criteria for 1.9\%, 3.9\% and 2.9\% of patients treated with FOR $12 \mu \mathrm{g}$, FP/FOR 250/10 $\mu \mathrm{g}$ or FP/FOR 500/20 $\mu$ g, respectively, radiological confirmation alone led to a slight decrease in reported pneumonia incidence in all treatment groups to $1.5 \%, 3.2 \%$ and $2.4 \%$, respectively [24]. Similarly, in two 1-year trials that compared the efficacy and safety of FF/VI versus VI, incidences of pneumonia events with compatible parenchymal infiltrates shown by chest radiograph, over-read by a central laboratory, were lower in all treatment groups (FF/VI 50/25 $\mu$ g 3.9\%; FF/VI 100/50 $\mu$ g 4.0\%; FF/VI 200/25 $\mu \mathrm{g} 4.6 \%$; and VI $25 \mu \mathrm{g}$ 1.8\%) compared with reported pneumonia defined as an AESI (5.9\%, 6.3\%, 6.8\% and 3.3\%, respectively) [46, 47]. The lower reported incidence of radiologically confirmed pneumonia in these studies, compared with investigator reporting alone, demonstrates the importance of accurate and consistent pneumonia reporting within these trials to allow evaluation of the incidence of pneumonia across different treatments. The reasons behind these observations are complex and multifactorial and could represent true misclassification, or could be due to factors such as availability of imaging and interpretation.

\section{Standardised symptoms and/or independent adjudication}

A further approach to define pneumonia is the use of a standardised list of clinical signs and symptoms, and this method has been used in several studies included in our review. Some of these studies also required treatment with antibiotics and/or antiviral and/or antifungal agents to define pneumonia, and/or adjudication by an independent clinical end-point committee. Such adjudication committees often have access to a patient's full medical record and may, in some cases, adjudicate an event as pneumonia even if the initial chest radiograph was clear.

In the RISE study, confirmed pneumonias were defined by the presence of a new infiltrate on a chest radiography as well as evidence of two or more of a standardised list of clinical signs and symptoms [33]. The reported incidence of pneumonia was $0.5 \%(n=3)$ of patients treated with budesonide (BUD)/FOR and $1.0 \%(n=6)$ of patients treated with FOR alone [33]. In the SOPHOS study, all potential pneumonia cases were adjudicated by an external clinical end-point committee [31]. Pneumonia was reported for $1.6 \%$ $(n=10)$ and $2.4 \%(n=15)$ of patients treated with BUD/FOR 320/10 $\mu$ g and 160/10 $\mu$ g, respectively, compared with $2.3 \%(n=14)$ of patients treated with FOR $10 \mu \mathrm{g}$ [31]. The phase III ETHOS and KRONOS studies, which investigated single-inhaler triple therapy with BUD/GLY/FOR versus BUD/FOR and GLY/FOR, required clinical diagnosis by the investigator, compatible chest imaging, two or more of a standardised list of clinical signs, symptoms or laboratory findings and treatment with antibiotics and/or antiviral and/or antifungal agents as part of the definition of pneumonia [14, 26]. All pneumonia adverse events in the ETHOS and KRONOS studies were adjudicated by an independent clinical end-point committee. Across these studies, confirmation of initial reported pneumonias by the independent clinical end-point committee led to a reduction in the reported rates (table 2) [14, 26]. In the TELOS study, pneumonia was defined according to clinical diagnosis by the investigator alongside compatible chest imaging, treatment with antibiotics and/or appropriate antiviral or antifungal agents, and two or more of a list of respiratory symptoms [32]. Similar to ETHOS and KRONOS, an independent clinical end-point committee reviewed all adverse events reported as pneumonia. The incidence of pneumonia was low, with adjudicated pneumonia reported for $0.8-1.4 \%$ of patients treated with BUD/FOR. Overall, these studies further demonstrate the importance of taking into account the way that pneumonia events are reported when reviewing pneumonia data from COPD clinical trials.

\section{Real-world evidence}

Our literature search also identified two real-world evidence studies reporting the incidence of pneumonia in patients treated with ICS. Notably, both reported the incidence of serious pneumonia events rather than 
TABLE 2 Pneumonia incidence determined by clinical end-point committee

\begin{tabular}{|c|c|c|c|}
\hline Study and treatment group & Patients, $n$ & $\begin{array}{l}\text { Pneumonia events submitted to clinical } \\
\text { end-point committee, } n(\%)\end{array}$ & $\begin{array}{c}\text { Pneumonia events confirmed by clinical } \\
\text { end-point committee, } \mathrm{n}(\%)\end{array}$ \\
\hline \multicolumn{4}{|l|}{ ETHOS [14] } \\
\hline BUD/GLY/FOR (160/18/9.6 $\mu \mathrm{g})$ & 2124 & $100(4.7)$ & $75(3.5)$ \\
\hline BUD/GLY/FOR $(320 / 18 / 9.6 \mu \mathrm{g})$ & 2144 & $115(5.4)$ & $90(4.2)$ \\
\hline BUD/FOR $(320 / 9.6 \mu \mathrm{g})$ & 2136 & $118(5.5)$ & $96(4.5)$ \\
\hline GLY/FOR $(18 / 9.6 \mu \mathrm{g})$ & 2125 & $66(3.1)$ & $48(2.3)$ \\
\hline \multicolumn{4}{|l|}{ KRONOS [26] } \\
\hline BUD/GLY/FOR (320/18/9.6 g; via MDI) & 639 & $16(2.5)$ & $12(1.9)$ \\
\hline BUD/FOR (320/9.6 $\mu \mathrm{g} ;$ via MDI) & 314 & $7(2.2)$ & $6(1.9)$ \\
\hline BUD/FOR (400/12 $\mu \mathrm{g} ;$ via DPI) & 318 & $6(1.9)$ & $4(1.3)$ \\
\hline GLY/FOR (18/9.6 нg; via MDI) & 625 & $11(1.8)$ & $10(1.6)$ \\
\hline
\end{tabular}

all pneumonia, as the study protocol restricted the collection of safety data to serious events only, with SuIssa et al. [37] defining serious pneumonia as incidences of pneumonia requiring hospitalisation, and Vestвo et al. [40,62] defining it as the proportion of participants that experienced pneumonia that resulted in death, was life threatening, required hospitalisation or prolongation of hospitalisation. These events have also been considered a significant medical event in the investigator's judgement. SuISSA et al. [37] performed a retrospective, observational cohort study to evaluate the effectiveness of ICS/LABA versus LAMA in patients with COPD using the UK Clinical Practice Research Datalink and propensity score matching. The study reported annual rates of hospital admission due to serious pneumonia of 7.6 and 5.2 per 100 person-years, respectively [37]. The Salford Lung Study evaluated the effectiveness of FF/VI in clinical practice, and reported pneumonia serious adverse events (SAEs) as part of its safety assessments by means of monitoring of electronic health records [40]. The study reported pneumonia SAEs in $6.7 \%$ $(n=94)$ of patients on FF/VI versus $5.9 \%(n=83)$ of patients who continued usual care across a 1-year period [40]. However, information on how these cases were diagnosed is not provided in the publication. Additionally, caution should be taken in the interpretation of these results, as pneumonia events were reported based on the randomised treatment arm and while patients randomised to FF/VI were allowed to switch to usual care, switching from usual care to FF/VI was not permitted [40].

It should also be noted that the accuracy of pneumonia as a clinically coded diagnosis in usual clinical practice is low. Studies performed in UK hospitals have shown that up to $50 \%$ of all diagnoses in discharge summaries are inaccurate and $>30 \%$ of pneumonia clinical coding is unreliable [63-65]. In these studies, $27-47 \%$ of coded pneumonia diagnoses did not have any evidence of consolidation in chest radiographs [63, 64], suggesting that clinical coding alone may be overestimating the incidence of pneumonia. This supports the lower incidence of pneumonia seen in studies that used chest radiography or adjudication to confirm pneumonia events, and also highlights a need to improve pneumonia diagnoses within usual clinical practice in addition to clinical studies.

\section{Distinguishing between pneumonia and COPD exacerbations}

A key clinical challenge regarding pneumonia capture in patients with COPD is distinguishing between pneumonia and COPD exacerbations due to the overlap in clinical presentation [8]. COPD trials evaluating ICS-containing therapy are likely to have a patient population that is predisposed to exacerbations and are often enriched for this trait, as the addition of ICS treatment is recommended for patients with COPD with persistent exacerbations despite long-acting bronchodilator therapy [9]. During an exacerbation, chest radiographs may demonstrate pulmonary infiltrates [7], potentially resulting in an increase in the reported rate of pneumonia in the absence of elevated white blood cell count or fever. In two replicate 1-year trials comparing treatment with FF/VI versus VI alone, pneumonia was reported as an adverse event for only 72 (60\%) of the 120 exacerbation events for which an infiltrate was detected via chest radiography, demonstrating that pneumonia may go unreported in the context of a COPD exacerbation event [47]. This clinical overlap of symptoms observed between COPD exacerbations and pneumonia, as well as the potential for patients to experience an exacerbation in the presence of comorbid pneumonia, poses a further challenge for the clinical diagnosis of pneumonia [66]. In addition, treatment decisions are not a reliable criterion for distinguishing between a pneumonia event and an exacerbation, as both are treated with antibiotics [67]. Furthermore, these patients are also often treated with systemic corticosteroids, which are 
known to elevate white blood cells and suppress fever upon initiation and could further confuse the distinction between pneumonia and an exacerbation [68]. There are regional cultural differences in acceptability of the term exacerbation versus pneumonia, and diagnosis may influence access to antibiotics [69-71]. Differentiation is also confounded by an association between unresolved exacerbations and pneumonia, which has been reported for FP treatment [57]. Finally, patients with COPD, particularly those with severe disease, may be at risk of cardiac and other pulmonary conditions that may be misclassified as exacerbations or pneumonia [72]. As such, the accurate capture of pneumonia is further complicated by the clinical characteristics of COPD.

Differences in study design and population characteristics While the method used for diagnosing pneumonia can affect the reported incidence in COPD trials, this is not the only aspect of a study that can affect pneumonia incidence rates. Particulars of the study design, such as the length of the study, as well as the characteristics and demographics of the patients enrolled can play an important part in the reported incidence of pneumonia.

\section{Study design}

Study design is likely to contribute to the varied reported incidences of pneumonia across the included studies. For example, the TORCH study had a 3-year treatment period and the INSPIRE study had a 2-year treatment period [56, 58], whereas SUMMIT was event driven with a median treatment duration of 1.8 years [38]. Other studies such as KRONOS and SUNSET were 24 or 26 weeks long (table 1) [26, 27]. With an event occurring as infrequently as pneumonia, these differences in study length impact the proportion of patients who experience the event during the course of the study. In addition, pneumonia has been shown to follow seasonal patterns, with relatively high pneumonia rates reported in winter; therefore, seasonality probably contributes to differences in pneumonia incidence according to study timing and length [73]. Furthermore, elements such as the design of the period prior to initiating study treatment are likely to affect the risk of patients experiencing pneumonia. For example, patients in IMPACT continued on their own medication for 2 weeks prior to initiating study treatment [13], whereas patients in the FLAME trial had a 4-week run-in period during which their own medication, including ICS, was stopped, and they received daily tiotropium (TIO) treatment, potentially removing patients from the trial who were most likely to benefit from ICS [34]. These elements of the study design are important considerations when interpreting the reported pneumonia incidence.

\section{Population characteristics}

A number of risk factors for pneumonia have been identified for patients with COPD, including older age, prior COPD exacerbation or respiratory tract infection, low body mass index, dyspnoea, presence of bronchiectasis or history of asthma, history of pneumonia, low blood eosinophil count, active smoking and severe airflow limitation [6, 20, 59, 73, 74]. A recent meta-analysis reported a significant difference in the risk of pneumonia according to the severity of COPD [21]. An increased incidence of investigator-reported pneumonia in patients in Asia compared with those in non-Asia regions was reported in the IMPACT study [75]. Pneumonia may be more common in Asian patients; however, differences in diagnostic processes, with more events diagnosed with chest radiography or computed tomography in Asia, may also contribute to the differences in pneumonia rates [75]. Inclusion of patients with these characteristics in study populations is highly likely to contribute to the variation in the reported rates of pneumonia between studies.

\section{Exacerbation history}

The phase III KRONOS and ETHOS studies both investigated single-inhaler triple therapy with BUD/ GLY/FOR versus BUD/FOR and GLY/FOR and used the same set of criteria for pneumonia capture and assessment. However, the two studies had different inclusion criteria, and therefore different patient populations. Patients enrolled in the 24-week KRONOS study were not required to have experienced a COPD exacerbation and overall 74\% ( $\mathrm{n}=1411)$ of patients experienced no moderate/severe exacerbations in the year prior to screening [26]. The risk of adjudicated pneumonia events with BUD/GLY/FOR 320/18/

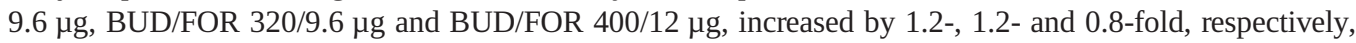
versus GLY/FOR 18/9.6 $\mu$ g treatment (table 1) [26]. In contrast, in the 52-week ETHOS study patients were required to have experienced at least one moderate/severe COPD exacerbation (if forced expiratory volume in $1 \mathrm{~s}\left(\mathrm{FEV}_{1}\right)<50 \%$ predicted) or at least two moderate or at least one severe exacerbation (if $\mathrm{FEV}_{1} \geqslant 50 \%$ pred $)$ in the year prior to screening [14]. In total, $57 \%(\mathrm{n}=4810)$ of patients experienced two or more moderate/severe exacerbations and $21 \%(n=1801)$ of patients experienced one or more severe exacerbations in the year before screening [14]. Risk of adjudicated pneumonia events increased by 1.9-, 1.6- and 2.0-fold in patients treated with BUD/GLY/FOR 320/18/9.6 $\mu \mathrm{g}$, BUD/GLY/FOR 160/18/9.6 $\mu \mathrm{g}$ and BUD/FOR 320/9.6 $\mu \mathrm{g}$, respectively, versus GLY/FOR 18/9.6 $\mu \mathrm{g}$ (table 1) [14]. This increased risk of 
pneumonia with ICS- versus non-ICS-containing treatment versus those reported for the low-exacerbating KRONOS population support the contribution of a prior history of exacerbation to varying pneumonia incidence across studies.

Similarly, other studies conducted in high-exacerbating populations have reported higher incidences of pneumonia than those including low-exacerbating populations. The LANTERN study enrolled patients with a history of one or fewer moderate/severe exacerbation in the previous year [43]. The incidence of investigator-reported pneumonia was 3.4-fold higher in patients treated with FP/SAL $(2.7 \%, \mathrm{n}=10)$ versus IND/GLY $(0.8 \%, n=3)$ [43]. The ILLUMINATE and INSTEAD studies required patients to have experienced no moderate/severe exacerbations in the year prior to screening [36, 44]. These studies reported very low incidences of pneumonia: $1.5 \%(n=4)$ of patients treated with FP/SAL and no patients treated with IND/GLY in ILLUMINATE reported radiographically confirmed pneumonia; $0.7 \%$ ( $n=2$ ) of patients treated with FP/SAL and no patients treated with IND in INSTEAD reported pneumonia SAEs [36, 44]. In contrast, ОHAR et al. [35] conducted a randomised, parallel-group study comparing FP/SAL with SAL monotherapy for the treatment of patients with COPD who had experienced an exacerbation within the previous 14 days. Treatment with FP/SAL was associated with a 1.3-fold increased risk of radiographically confirmed pneumonia versus SAL, with pneumonia reported for $4.1 \%(n=13)$ and $3.1 \%$ $(n=10)$ of patients, respectively [35].

\section{Lung function}

The identified studies recruited patients with a wide range of airflow limitation severity. Overall, the literature search identified 12 studies that recruited only patients with severe airflow limitation (post-bronchodilator $\mathrm{FEV}_{1} \leqslant 50 \%$ pred) [10-12, 24, 28, 29, 45, 52-56]. The incidence of pneumonia for patients treated with ICS-containing therapies in these studies ranged from $0.4 \%$ to $7.6 \%$, while the incidence for patients treated with non-ICS-containing therapies in these studies ranged from $0.3 \%$ to $5.5 \%$ [10-12, 24, 28, 29, 45, 52-56].

The SUNSET study recruited patients with moderate to severe airflow limitation (post-bronchodilator $\mathrm{FEV}_{1} \geqslant 40 \%$ pred and $<80 \%$ pred), with a mean $\mathrm{FEV}_{1}$ of $57 \%$ pred at baseline [27]. Pneumonia was reported for a relatively small proportion of patients: $1.7 \%(n=9)$ of patients treated with triple therapy with FP/SAL and TIO and 1.1\% ( $n=6)$ of patients treated with IND/GLY [27]. This represents a 1.5-fold increased risk of pneumonia with ICS/LABA/LAMA versus LABA/LAMA treatment in this study. The ILLUMINATE trial also included patients with a post-bronchodilator $\mathrm{FEV}_{1}$ of $40-80 \%$ pred [36]. The study population had a mean $\mathrm{FEV}_{1}$ of $51 \%$ pred at baseline, and a relatively small proportion of patients experienced radiologically confirmed pneumonia (FP/SAL: 1.5\%, $n=4$; IND/GLY: 0) [36]. By comparison, in patients from the ETHOS study, who had a post-bronchodilator $\mathrm{FEV}_{1}$ of $43.1-43.6 \%$ pred, confirmed pneumonia was reported in $3.5-4.5 \%$ of patients receiving ICS during the study versus $2.3 \%$ for patients not receiving ICS [14]. These relatively high proportions of patients with confirmed pneumonia in the ETHOS study may reflect the relatively high proportion of patients who had severe airflow limitation, as $\sim 70 \%$ of patients had a post-bronchodilator $\mathrm{FEV}_{1}<50 \%$ pred [14]. The SUMMIT study recruited only patients with moderate airflow limitation $\left(\mathrm{FEV}_{1} \geqslant 50 \%\right.$ pred and $\leqslant 70 \%$ pred), yet reported pneumonia in a relatively high proportion of patients: 5.7\% $(n=237), 5.5 \%(n=228), 3.9 \%(n=163)$ and 5.2\% $(n=214)$ of patients treated with FF/VI, FF/VI, VI and placebo, respectively. However, it is worth noting that SUMMIT recruited patients with a history, or at increased risk, of cardiovascular disease and the mean study exposure was 1.8 years, which may account for the difference observed between the SUMMIT and ETHOS trials. These studies highlight the complexities of measuring the incidence of pneumonia and demonstrate the need to consider all study characteristics when interpreting the findings with regard to pneumonia incidence in different trials.

Towards a standardised definition of pneumonia for COPD clinical trials

This review has shown that pneumonia in COPD trials has been captured using a variety of methods, including investigator reporting of pneumonia adverse events or confirmation with radiographic imaging with or without the requirement for specific clinical symptoms or laboratory findings, antibiotic and/or antiviral and/or antifungal treatment, or adjudication by an independent committee. Therefore, meaningful comparisons of pneumonia rates between individual trials cannot be made. Furthermore, these results can only be interpreted with knowledge of the study design, patient population, the countries where the study was based and how the diagnosis of pneumonia was made. This adds a burden to readers who may choose to accept a figure given in an abstract rather than look further.

We propose that a minimal set of standardised criteria for the diagnosis of pneumonia should be used in COPD clinical trials that include pneumonia as an outcome or as an expected adverse event 
(supplementary table S1). This would allow comparison of pneumonia rates between COPD trials, which is of critical importance as pneumonia is a known safety signal for ICS-containing treatments. As mentioned previously, MedDRA has recently developed a Standardised MedDRA Query for Infective Pneumonia that could be used to standardise the capture and analysis of pneumonia-related events in clinical trials [25]. As history, physical examination and laboratory data are inadequate to either exclude or diagnose community-acquired pneumonia [76], suspected pneumonia should be confirmed using a chest radiograph or computed tomography scan. Diagnostic criteria as outlined by the British Thoracic Society, which are straightforward and emanate from an authoritative professional society, would make a good base for standardisation [77], while a requirement for all pneumonia cases to be adjudicated by an independent committee with explicitly agreed-upon criteria would also improve the ability to compare pneumonia incidences between studies. Furthermore, the history of pneumonia within the patient population at baseline should be reported for all trials as well as the criteria for the diagnosis of pneumonia. In addition, the use of individual participant data from randomised controlled COPD trials could be used to derive standardised outcome definitions across trials [78]. It should be reiterated that this set of recommendations refers to the diagnosis of pneumonia in COPD clinical trials and not the diagnosis of pneumonia in usual clinical practice. However, our recommendations could be extended beyond pneumonia in patients with COPD and applied to any clinical trial in which pneumonia is a reported outcome.

\section{Conclusions}

Pneumonia is a known class effect of ICS in patients with COPD [19]. Clinical trials that have examined the long-term use of ICS in patients with COPD have reported large differences in the rates of pneumonia. Differences in the prevalence of risk factors for pneumonia between study populations may contribute to this variation, and to the ability to detect differences in pneumonia rates and their magnitude. Furthermore, analysis of pneumonia as a group of pneumonia-related terms and less stringent confirmation criteria may lead to relatively greater reported rates of pneumonia than the use of a narrower set of terms and adjudication by an independent clinical end-point committee. Across-study comparisons of pneumonia incidence are problematic and the potential confounding factors, such as population risk of pneumonia and variation in pneumonia definitions, should be carefully considered. Therefore, across-study comparisons of pneumonia incidence should be avoided. Greater transparency in the reporting of the methods used to define pneumonia is critical to allow pneumonia rates to be evaluated in the context of other studies. As such, within-trial comparisons of ICS-containing versus non-ICS-containing treatments are the only appropriate method to assess the influence of ICS on pneumonia incidence. Importantly, evaluation of the overall riskbenefit profile of the use of ICS in patients with COPD should include the impact on exacerbation risk, lung function, health-related quality of life and mortality as well as the incidence of pneumonia.

A minimal, standardised set of criteria for the diagnosis of pneumonia should be used in studies with pneumonia as an outcome or as an expected adverse event. The criteria used should be clearly defined in the study protocol and the resulting publication.

Provenance: Submitted article, peer reviewed.

Acknowledgements: Editorial support in the form of collating search results, writing assistance, assembling figures, collating author comments, grammatical editing and referencing was provided by Anne Errichelli, Fishawack Indicia Ltd, UK, part of Fishawack Health, and was funded by GlaxoSmithKline (GSK). Dave Singh is supported by the National Institute for Health Research Manchester Biomedical Research Centre.

Author contributions: David A. Lipson contributed to the conception/design of this analysis. Gerard J. Criner, David M.G. Halpin and Mark T. Dransfield were involved in the acquisition of the data. All authors were involved in the analysis and interpretation of the data and editing of the article and approved the final version of the manuscript before submission.

Conflict of interest: R.A. Wise reports other and non-financial support from GlaxoSmithKline (funding the study and funding medical writing support by Anne Errichelli at Fishawack Indicia Ltd, UK), during the conduct of the study; grants and personal fees from AstraZeneca/Medimmune/Pearl (data monitoring committee, grants, consulting), grants and personal fees from Boehringer Ingelheim (steering committee, data monitoring committee, grants), personal fees from Contrafect (clinical end-point committee), grants and personal fees from AstraZeneca (research grant, consulting), grants and personal fees from GSK (research grant, consulting), personal fees from Sunovion (workshop, consulting), personal fees from Merck (data monitoring committee), personal fees from Verona (consultant), personal fees from Mylan/Theravance (consultant), personal fees from Propeller Health (consultant), grants and personal fees from GSK (scientific advisory board, clinical end-point committee, Data Monitoring Committee, research grant support), personal fees from Novartis (consultant), personal fees from ChimRix (data 
monitoring committee), personal fees from FSD Pharma (data monitoring committee), personal fees from AbbVie (data monitoring committee), personal fees from Bristol Myers Squibb (data monitoring committee), personal fees from Puretech (data monitoring committee), personal fees from Galderma (clinical end-point committee), personal fees from Chiesi (clinical end-point committee), outside the submitted work. M. Bafadhel reports other and non-financial support from GlaxoSmithKline (funding the study and funding medical writing support by Anne Errichelli at Fishawack Indicia Ltd, UK), during the conduct of the study; grants, personal fees and other from AstraZeneca (research grants, advisory board attendance, educational meeting attendance), personal fees and other from Chiesi (advisory board attendance, educational meeting attendance), personal fees and other from Boehringer Ingelheim (advisory board attendance, educational meeting attendance), personal fees and other from GlaxoSmithKline (advisory board attendance), personal fees and other from ProAxsis (scientific advisor), personal fees and other from AlbusHealth (scientific advisor), outside the submitted work. C. Crim reports other and non-financial support from GlaxoSmithKline (funding the study and funding medical writing support by Anne Errichelli at Fishawack Indicia Ltd, UK), during the conduct of the study; C. Crim is a former employee of GlaxoSmithKline and has shares/options held in GSK, outside the submitted work. G.J. Criner reports other and non-financial support from GlaxoSmithKline (funding the study and funding medical writing support by Anne Errichelli at Fishawack Indicia Ltd, UK), during the conduct of the study; personal fees from Almirall, AstraZeneca, Boehringer Ingelheim, Chiesi, CSA Medical, Eolo, GlaxoSmithKline, HGE Technologies, Novartis, Nuvaira, Olympus, Pulmonx and Verona, outside the submitted work. N.C. Day reports other and non-financial support from GlaxoSmithKline (funding the study and funding medical writing support by Anne Errichelli at Fishawack Indicia Ltd, UK), during the conduct of the study; N.C. Day is an employee of GlaxoSmithKline and has shares/options held in GSK, outside the submitted work. D.M.G. Halpin reports other and non-financial support from GlaxoSmithKline (funding the study and funding medical writing support by Anne Errichelli at Fishawack Indicia Ltd, UK), during the conduct of the study; personal fees from AstraZeneca, personal fees and non-financial support from Boehringer Ingelheim, personal fees from Chiesi, personal fees from GlaxoSmithKline, personal fees and non-financial support from Novartis, personal fees from Pfizer, outside the submitted work. M.K. Han reports other and non-financial support from GlaxoSmithKline (funding the study and funding medical writing support by Anne Errichelli at Fishawack Indicia Ltd, UK), during the conduct of the study; outside the submitted work, M.K. Han also reports personal fees from GlaxoSmithKline, AstraZeneca, Boehringer Inhgelheim, Cipla, Chiesi, Novartis, Pulmonx, Teva, Verona, Merck, Mylan, Sanofi, DevPro, Aerogen, Polarian, Regeneron, United Therapeutics, Medscape and Integrity. She has received either in kind research support or funds paid to the institution from the $\mathrm{NIH}$, Novartis, Sunovion, Nuvaira, Sanofi, AstraZeneca, Boehringer Ingelheim, Gala Therapeutics, Biodesix, the COPD Foundation and the American Lung Association. She has participated in Data Safety Monitoring Boards for Novartis and Medtronic with funds paid to the institution. P. Lange reports other and non-financial support from GlaxoSmithKline (funding the study and funding medical writing support by Anne Errichelli at Fishawack Indicia Ltd, UK), during the conduct of the study; personal fees from AstraZeneca, grants and personal fees from Boehringer Ingelheim, personal fees from GlaxoSmithKline, outside the submitted work. D.A. Lipson reports other and non-financial support from GlaxoSmithKline (funding the study and funding medical writing support by Anne Errichelli at Fishawack Indicia Ltd, UK), during the conduct of the study; D.A. Lipson is an employee of GlaxoSmithKline and has shares/options held in GSK, outside the submitted work. F.J. Martinez reports other and non-financial support from GlaxoSmithKline (funding the study and funding medical writing support by Anne Errichelli at Fishawack Indicia Ltd, UK), during the conduct of the study; other from Afferent/Merck (IPF Steering Committee (academic productivity)), personal fees, non-financial support and other from AstraZeneca (COPD Advisory Boards (personal fees honoraria and non-personal travel support), study steering committee (non-personal travel support), DSMB (other - academic co-authorship)), other from Bayer (ILD Steering Committee (academic productivity)), personal fees, non-financial support and other from Boehringer Ingelheim (COPD Advisory Board (personal fees and non-personal travel support); ATS presentation (personal fees); progressive pulmonary fibrosis DSMB (no financial support but travel); ERS IPF study result presentation (personal fee and travel support; IPF study steering committee chair), non-financial support and other from Bioscale/ProterrixBio (COPD scientific advisory board (no direct financial compensation, support for NIH study)), other from Bridge Biotherapeutics (IPF consultation), personal fees and non-financial support from Canadian Respiratory Network (COPD CME presentation (honorarium and travel support)), personal fees and non-financial support from Chiesi (COPD CME presentation (personal fees honoraria and non-personal travel support); Advisory Board (personal fees honoraria and travel support)), personal fees from France Foundation (IPF CME presentation (honorarium)), personal fees and non-financial support from Genentech (COPD advisory board (personal fee and non-personal travel support) and asthma DSMB (no support); IPF advisory board (personal fees honorarium and non-personal travel support)), other from Gilead (IPF study steering committee (other - co-authorship)), personal fees and non-financial support from GlaxoSmithKline (COPD advisory boards (personal fees honoaria and non-personal travel support), study steering committee (non-personal travel support), DSMB (other - academic co-authorship)), personal fees and non-financial support from Inova Fairfax Health System (COPD CME presentation (personal support honorarium and non-personal travel support)), personal fees from MD Magazine (COPD CME programme (personal fee honorarium and non-personal travel support)), personal fees and non-financial support from 
Methodist Hospital Brooklyn (IPF and COPD CME programs (personal fee honoraria)), personal fees and non-financial support from Miller Communications (COPD and IPF CME programs (personal fees honoraria and non-personal travel support)), non-financial support from Nitto (IPF Study Teleconference and Steering Committee (non-personal travel support)), personal fees and non-financial support from Novartis (COPD advisory board and international meeting COPD disease presentations (personal fees honoraria and non-personal travel support)), personal fees from New York University (ILD CME programme (personal fee honoraria)), personal fees and other from Patara (venture capital expert advice for IPF study (personal fees honoraria); IPF steering committee), personal fees and non-financial support from Pearl Pharmaceuticals (COPD advisory boards (personal fee honoraria and non-personal travel support) and COPD steering committee (academic productivity)), personal fees and non-financial support from PeerView Communications (COPD and IPF CME programmes (personal fees honoraria and non-personal travel support)), personal fees, non-financial support and other from Physicians Education Resource (IPF advisory board (personal fee honorarium and travel support)), personal fees from Prime Communications (COPD CME programme (personal fee honorarium)), other from Roche (IPF Steering Committee (academic productivity)), other from ProMetic (IPF steering committee (academic productivity)), personal fees from Rare Disease Health Communications (IPF CME programme (personal fee honorarium)), personal fees from Rockpointe (COPD CME programme (personal fee honorarium)), other from Biogen (IPF study DSMB (no support) and IPF study steering committee (academic productivity)), personal fees and non-financial support from Sunovion (COPD advisory boards (personal fee honoraria and non-personal travel support)), personal fees and non-financial support from Teva (COPD advisory board (personal fee honorarium and travel support)), personal fees and non-financial support from University of Alabama Birmingham (IPF CME presentation (personal fee honoraria and non-personal travel support)), personal fees from UpToDate (COPD CME (personal fee honoraria)), other from Veracyte (IPF study steering committee (other co-authorship)), personal fees from WebMD/MedScape (COPD and IPF CME presentations (personal fee honoraria)), non-financial support from Zambon (IPF study meeting (non-personal travel support) and advisory board (personal fees honorarium)), personal fees and non-financial support from American College of Chest Physicians, personal fees and non-financial support from ConCert, personal fees and non-financial support from Continuing Education, personal fees and non-financial support from National Society for Continuing Education, personal fees and non-financial support from Potomac, personal fees and non-financial support from Puerto Rico Respiratory Society, personal fees and non-financial support from Theravance, non-financial support from Nitto, personal fees from Columbia University, personal fees from Integras, personal fees from Unity, personal fees from Western Connecticut Health Network, personal fees from Academic CME, personal fees from Platform IQ, personal fees from American Thoracic Society, grants from Promedior, outside the submitted work. D.J. Maselli reports other and non-financial support from GlaxoSmithKline (funding the study and funding medical writing support by Anne Errichelli at Fishawack Indicia Ltd, UK), during the conduct of the study; personal fees from AstraZeneca, personal fees from Sanofi/Regeneron, personal fees from Amgen, outside the submitted work. D. Midwinter reports other and non-financial support from GlaxoSmithKline (funding the study and funding medical writing support by Anne Errichelli at Fishawack Indicia Ltd, UK), during the conduct of the study; D. Midwinter is an employee of GlaxoSmithKline and has shares/options held in GSK, outside the submitted work. D. Singh reports other and non-financial support from GlaxoSmithKline (funding the study and funding medical writing support by Anne Errichelli at Fishawack Indicia Ltd, UK), during the conduct of the study; personal fees from GlaxoSmithKline, grants and personal fees from AstraZeneca, grants and personal fees from Boehringer Ingelheim, grants and personal fees from Chiesi, personal fees from Cipla, personal fees from Genentech, grants and personal fees from Glenmark, grants and personal fees from Menarini, grants and personal fees from Mundipharma, grants and personal fees from Novartis, personal fees from Peptinnovate, grants and personal fees from Pfizer, grants and personal fees from Pulmatrix, grants and personal fees from Theravance, grants and personal fees from Verona, outside the submitted work. M. Zysman reports other and non-financial support from GlaxoSmithKline (funding the study and funding medical writing support by Anne Errichelli at Fishawack Indicia Ltd, UK), during the conduct of the study; grants and personal fees from AstraZeneca, grants and personal fees from Boehringer Ingelheim, personal fees from Novartis, personal fees from Chiesi, personal fees from GlaxoSmithKline, personal fees from CSL Behring, outside the submitted work. M.T. Dransfield reports other and non-financial support from GlaxoSmithKline (funding the study and funding medical writing support by Anne Errichelli at Fishawack Indicia Ltd, UK), during the conduct of the study; other from Boehringer Ingelheim (consulting and contracted clinical trials), personal fees and other from GlaxoSmithKline (consulting and contracted clinical trials), personal fees and other from AstraZeneca (consulting and contracted clinical trials), other from PneumRx/BTG (contracted clinical trials), other from Pulmonx (contracted clinical trials), personal fees from Teva (consulting), other from Gala (contracted clinical trials), other from Nuvaira (contracted clinical trials), outside the submitted work. R.E.K. Russell reports other and non-financial support from GlaxoSmithKline (funding the study and funding medical writing support by Anne Errichelli at Fishawack Indicia Ltd, UK), during the conduct of the study; grants and personal fees from AstraZeneca, personal fees from GlaxoSmithKline, personal fees from Cipla, personal fees from Chiesi, personal fees from Boehringer Ingelheim, personal fees from Albus Health (Advisor), non-financial support from National Institute for Health Research Oxford Biomedical Research Centre, outside the submitted work. 
Support statement: Pharmaceutical company funded by GlaxoSmithKline. Funding information for this article has been deposited with the Crossref Funder Registry.

\section{References}

$1 \quad$ Brown JS. Community-acquired pneumonia. Clin Med 2012; 12: 538-543.

2 Torres A, Cilloniz C, Niederman MS, et al. Pneumonia. Nat Rev Dis Primers 2021; 7: 25.

3 Metlay JP, Waterer GW, Long AC, et al. Diagnosis and treatment of adults with community-acquired pneumonia. An official clinical practice guideline of the American Thoracic Society and Infectious Diseases Society of America. Am J Respir Crit Care Med 2019; 200: e45-e67.

4 Almirall J, Serra-Prat M, Bolíbar I, et al. Risk factors for community-acquired pneumonia in adults: a systematic review of observational studies. Respiration 2017; 94: 299-311.

5 Jackson ML, Neuzil KM, Thompson WW, et al. The burden of community-acquired pneumonia in seniors: results of a population-based study. Clin Infect Dis 2004; 39: 1642-1650.

6 Müllerova H, Chigbo C, Hagan GW, et al. The natural history of community-acquired pneumonia in COPD patients: a population database analysis. Respir Med 2012; 106: 1124-1133.

7 Saleh A, López-Campos JL, Hartl S, et al. The effect of incidental consolidation on management and outcomes in COPD exacerbations: data from the European COPD Audit. PLoS One 2015; 10: e0134004.

8 Lieberman D, Lieberman D, Gelfer Y, et al. Pneumonic vs nonpneumonic acute exacerbations of COPD. Chest 2002; 122: 1264-1270.

9 Global Initiative for Chronic Obstructive Lung Disease (GOLD). Global Strategy for the Diagnosis, Management and Prevention of COPD. 2021. Available from: https://goldcopd.org/ Date last accessed: December 2020.

10 Anzueto A, Ferguson GT, Feldman G, et al. Effect of fluticasone propionate/salmeterol (250/50) on COPD exacerbations and impact on patient outcomes. COPD 2009; 6: 320-329.

11 Ferguson GT, Anzueto A, Fei R, et al. Effect of fluticasone propionate/salmeterol (250/50 microg) or salmeterol (50 microg) on COPD exacerbations. Respir Med 2008; 102: 1099-1108.

12 Papi A, Vestbo J, Fabbri L, et al. Extrafine inhaled triple therapy versus dual bronchodilator therapy in chronic obstructive pulmonary disease (TRIBUTE): a double-blind, parallel group, randomised controlled trial. Lancet 2018; 391: 1076-1084.

13 Lipson DA, Barnhart F, Brealey N, et al. Once-daily single-inhaler triple versus dual therapy in patients with COPD. N Engl J Med 2018; 378: 1671-1680.

14 Rabe KF, Martinez FJ, Ferguson GT, et al. Triple inhaled therapy at two glucocorticoid doses in moderate-to-very-severe COPD. N Engl J Med 2020; 383: 35-48.

15 Ye W, Guo X, Yang T, et al. Systematic review of inhaled corticosteroid withdrawal effects in chronic obstructive pulmonary disease, and comparison with two 'real-life' studies. J Thorac Dis 2018; 10: 4565-4573.

16 Kew KM, Seniukovich A. Inhaled steroids and risk of pneumonia for chronic obstructive pulmonary disease. Cochrane Database Syst Rev 2014; 3: CD010115.

17 Singh S, Amin AV, Loke YK. Long-term use of inhaled corticosteroids and the risk of pneumonia in chronic obstructive pulmonary disease: a meta-analysis. Arch Intern Med 2009; 169: 219-229.

18 Torén K, Blanc PD, Qvarfordt I, et al. Inhaled corticosteroids use and risk of invasive pneumococcal disease in a population-based study. Ann Am Thorac Soc 2020; 17: 1570-1575.

19 European Medicines Agency (EMA). Inhaled Corticosteroids Containing Medicinal Products Indicated in the Treatment of Chronic Obstructive Pulmonary Disease. 2016. www.ema.europa.eu/medicines/human/referrals/ inhaled-corticosteroids-containing-medicinal-products-indicated-treatment-chronic-obstructive/ Date last accessed: September 2020.

20 Zhang Q, Li S, Zhou W, et al. Risk of pneumonia with different inhaled corticosteroids in COPD patients: a meta-analysis. COPD 2020; 17: 462-469.

21 Chen $\mathrm{H}$, Sun J, Huang Q, et al. Inhaled corticosteroids and the pneumonia risk in patients with chronic obstructive pulmonary disease: a meta-analysis of randomized controlled trials. Front Pharmacol 2021; 12: 691621.

22 Miravitlles M, Auladell-Rispau A, Monteagudo M, et al. Systematic review on long-term adverse effects of inhaled corticosteroids in the treatment of COPD. Eur Respir Rev 2021; 30: 210075.

23 Kumamaru KK, Machitori A, Koba R, et al. Global and Japanese regional variations in radiologist potential workload for computed tomography and magnetic resonance imaging examinations. Jpn J Radiol 2018; 36: 273-281.

24 Papi A, Dokic D, Tzimas W, et al. Fluticasone propionate/formoterol for COPD management: a randomized controlled trial. Int J Chron Obstruct Pulmon Dis 2017; 12: 1961-1971.

25 Medical Dictionary [NLE4] for Regulatory Activities. Standardised MedDRA Queries. July 2021. Available from: https://admin.new.meddra.org/sites/default/files/guidance/file/000595_SMQ_intguide_24_1.pdf

26 Ferguson GT, Rabe KF, Martinez FJ, et al. Triple therapy with budesonide/glycopyrrolate/formoterol fumarate with co-suspension delivery technology versus dual therapies in chronic obstructive pulmonary disease (KRONOS): a double-blind, parallel-group, multicentre, phase 3 randomised controlled trial. Lancet Respir Med 2018; 6: 747-758. 
27 Chapman KR, Hurst JR, Frent SM, et al. Long-term triple therapy de-escalation to indacaterol/glycopyrronium in patients with chronic obstructive pulmonary disease (SUNSET): a randomized, double-blind, triple-dummy clinical trial. Am J Respir Crit Care Med 2018; 198: 329-339.

28 Magnussen H, Disse B, Rodriguez-Roisin R, et al. Withdrawal of inhaled glucocorticoids and exacerbations of COPD. N Engl J Med 2014; 371: 1285-1294.

29 Vestbo J, Papi A, Corradi M, et al. Single inhaler extrafine triple therapy versus long-acting muscarinic antagonist therapy for chronic obstructive pulmonary disease (TRINITY): a double-blind, parallel group, randomised controlled trial. Lancet 2017; 389: 1919-1929.

30 Jung KS, Park HY, Park SY, et al. Comparison of tiotropium plus fluticasone propionate/salmeterol with tiotropium in COPD: a randomized controlled study. Respir Med 2012; 106: 382-389.

31 Hanania NA, Papi A, Anzueto A, et al. Efficacy and safety of two doses of budesonide/formoterol fumarate metered dose inhaler in COPD. ERJ Open Res 2020; 6: 00187-2019.

32 Ferguson GT, Papi A, Anzueto A, et al. Budesonide/formoterol MDI with co-suspension delivery technology in COPD: the TELOS study. Eur Respir J 2018; 52: 1801334.

33 Ferguson GT, Tashkin DP, Skärby T, et al. Effect of budesonide/formoterol pressurized metered-dose inhaler on exacerbations versus formoterol in chronic obstructive pulmonary disease: the 6-month, randomized RISE (Revealing the Impact of Symbicort in reducing Exacerbations in COPD) study. Respir Med 2017; 132: 31-41.

34 Wedzicha JA, Banerji D, Chapman KR, et al. Indacaterol-glycopyrronium versus salmeterol-fluticasone for COPD. N Engl J Med 2016; 374: 2222-2234.

35 Ohar JA, Crater GD, Emmett A, et al. Fluticasone propionate/salmeterol 250/50 $\mu \mathrm{g}$ versus salmeterol $50 \mu \mathrm{g}$ after chronic obstructive pulmonary disease exacerbation. Respir Res 2014; 15: 105.

36 Vogelmeier CF, Bateman ED, Pallante J, et al. Efficacy and safety of once-daily QVA149 compared with twice-daily salmeterol-fluticasone in patients with chronic obstructive pulmonary disease (ILLUMINATE): a randomised, double-blind, parallel group study. Lancet Respir Med 2013; 1: 51-60.

37 Suissa S, Dell'Aniello S, Ernst P. Comparative effectiveness of LABA-ICS versus LAMA as initial treatment in COPD targeted by blood eosinophils: a population-based cohort study. Lancet Respir Med 2018; 6: 855-862.

38 Vestbo J, Anderson JA, Brook RD, et al. Fluticasone furoate and vilanterol and survival in chronic obstructive pulmonary disease with heightened cardiovascular risk (SUMMIT): a double-blind randomised controlled trial. Lancet 2016; 387: 1817-1826.

39 Crim C, Calverley PMA, Anderson JA, et al. Pneumonia risk with inhaled fluticasone furoate and vilanterol in COPD patients with moderate airflow limitation: the SUMMIT trial. Respir Med 2017; 131: 27-34.

40 Vestbo J, Leather D, Diar Bakerly N, et al. Effectiveness of fluticasone furoate-vilanterol for COPD in clinical practice. N Engl J Med 2016; 375: 1253-1260.

41 Vogelmeier C, Paggiaro PL, Dorca J, et al. Efficacy and safety of aclidinium/formoterol versus salmeterol/ fluticasone: a phase 3 COPD study. Eur Respir J 2016; 48: 1030-1039.

42 Zheng J, de Guia T, Wang-Jairaj J, et al. Efficacy and safety of fluticasone furoate/vilanterol (50/25 mcg; 100/ $25 \mathrm{mcg} ; 200 / 25 \mathrm{mcg}$ ) in Asian patients with chronic obstructive pulmonary disease: a randomized placebo-controlled trial. Curr Med Res Opin 2015; 31: 1191-1200.

43 Zhong N, Wang C, Zhou X, et al. LANTERN: a randomized study of QVA149 versus salmeterol/fluticasone combination in patients with COPD. Int J Chron Obstruct Pulmon Dis 2015; 10: 1015-1026.

44 Rossi A, van der Molen T, del Olmo R, et al. INSTEAD: a randomised switch trial of indacaterol versus salmeterol/fluticasone in moderate COPD. Eur Respir J 2014; 44: 1548-1556.

45 Wedzicha JA, Singh D, Vestbo J, et al. Extrafine beclomethasone/formoterol in severe COPD patients with history of exacerbations. Respir Med 2014; 108: 1153-1162.

46 Dransfield MT, Bourbeau J, Jones PW, et al. Once-daily inhaled fluticasone furoate and vilanterol versus vilanterol only for prevention of exacerbations of COPD: two replicate double-blind, parallel-group, randomised controlled trials. Lancet Respir Med 2013; 1: 210-223.

47 Crim C, Dransfield MT, Bourbeau J, et al. Pneumonia risk with inhaled fluticasone furoate and vilanterol compared with vilanterol alone in patients with COPD. Ann Am Thorac Soc 2015; 12: 27-34.

48 Kerwin EM, Scott-Wilson C, Sanford L, et al. A randomised trial of fluticasone furoate/vilanterol $(50 / 25 \mu \mathrm{g}$; 100/25 $\mu \mathrm{g}$ ) on lung function in COPD. Respir Med 2013; 107: 560-569.

49 Martinez FJ, Boscia J, Feldman G, et al. Fluticasone furoate/vilanterol (100/25; 200/25 $\mu \mathrm{g}$ ) improves lung function in COPD: a randomised trial. Respir Med 2013; 107: 560-569.

50 Doherty DE, Tashkin DP, Kerwin E, et al. Effects of mometasone furoate/formoterol fumarate fixed-dose combination formulation on chronic obstructive pulmonary disease (COPD): results from a 52-week phase III trial in subjects with moderate-to-very severe COPD. Int J Chron Obstruct Pulmon Dis 2012; 7: 57-71.

51 Tashkin DP, Doherty DE, Kerwin E, et al. Efficacy and safety of a fixed-dose combination of mometasone furoate and formoterol fumarate in subjects with moderate to very severe COPD: results from a 52-week phase III trial. Int J Chron Obstruct Pulmon Dis 2012; 7: 43-55.

52 Sharafkhaneh A, Southard JG, Goldman M, et al. Effect of budesonide/formoterol pMDI on COPD exacerbations: a double-blind, randomized study. Respir Med 2012; 106: 257-268. 
53 Calverley PM, Kuna P, Monsó E, et al. Beclomethasone/formoterol in the management of COPD: a randomised controlled trial. Respir Med 2010; 104: 1858-1868.

54 Rennard SI, Tashkin DP, McElhattan J, et al. Efficacy and tolerability of budesonide/formoterol in one hydrofluoroalkane pressurized metered-dose inhaler in patients with chronic obstructive pulmonary disease: results from a 1-year randomized controlled clinical trial. Drugs 2009; 69: 549-565.

55 Tashkin DP, Rennard SI, Martin P, et al. Efficacy and safety of budesonide and formoterol in one pressurized metered-dose inhaler in patients with moderate to very severe chronic obstructive pulmonary disease: results of a 6-month randomized clinical trial. Drugs 2008; 68: 1975-2000.

56 Wedzicha JA, Calverley PM, Seemungal TA, et al. The prevention of chronic obstructive pulmonary disease exacerbations by salmeterol/fluticasone propionate or tiotropium bromide. Am J Respir Crit Care Med 2008; 177: 19-26.

57 Calverley PMA, Stockley RA, Seemungal TAR, et al. Reported pneumonia in patients with COPD: findings from the INSPIRE study. Chest 2011; 139: 505-512.

58 Calverley PM, Anderson JA, Celli B, et al. Salmeterol and fluticasone propionate and survival in chronic obstructive pulmonary disease. N Engl J Med 2007; 356: 775-789.

59 Crim C, Calverley PM, Anderson JA, et al. Pneumonia risk in COPD patients receiving inhaled corticosteroids alone or in combination: TORCH study results. Eur Respir J 2009; 34: 641-647.

60 Berrington de González A, Darby S. Risk of cancer from diagnostic X-rays: estimates for the UK and 14 other countries. Lancet 2004; 363: 345-351.

61 GlaxoSmithKline. A Study Comparing the Efficacy, Safety and Tolerability of Fixed Dose Combination (FDC) of $\mathrm{FF} / \mathrm{UMEC} / \mathrm{VI}$ with the FDC of FF/VI and UMEC/VI; Administered Once-Daily via a Dry Powder Inhaler (DPI) in Subjects with Chronic Obstructive Pulmonary Disease (COPD). February 2021. www.gsk-studyregister.com/en/ trial-details/?id=116855/

62 GlaxoSmithKline. A Randomised Effectiveness Study Comparing Fluticasone Furoate (FF, GW685698)/ Vilanterol (VI, GW642444) With Standard Treatment in Chronic Obstructive Pulmonary Disease (COPD). https:// clinicaltrials.gov/ct2/show/NCT01551758/ Date last accessed: April 2021. Date last updated: 31 May 2017.

63 Pink K, Mitchell I, Davies H. P17 The accuracy of a diagnosis of pneumonia in a UK teaching hospital. Thorax 2012; 67: A71.

64 Tarpey D, Youzgin A. Accuracy of diagnosis of pneumonia in an UK hospital participating in the advancing quality initiative. Eur Respir J 2013; 42: P2708.

65 Tsopra R, Wyatt JC, Beirne P, et al. Level of accuracy of diagnoses recorded in discharge summaries: a cohort study in three respiratory wards. J Eval Clin Pract 2019; 25: 36-43.

66 Trethewey SP, Hurst JR, Turner AM. Pneumonia in exacerbations of COPD: what is the clinical significance? ERJ Open Res 2020; 6: 00282-2019.

67 Restrepo MI, Sibila O, Anzueto A. Pneumonia in patients with chronic obstructive pulmonary disease. Tuberc Respir Dis 2018; 81: 187-197.

68 Frenkel A, Kachko E, Novack V, et al. The association of glucocorticosteroid treatment with WBC count in patients with COPD exacerbation. J Clin Med 2019; 8: 1697.

69 Hurst JR. Consolidation and exacerbation of COPD. Med Sci 2018; 6: 44.

70 Siddiqi A, Sethi S. Optimizing antibiotic selection in treating COPD exacerbations. Int J Chron Obstruct Pulmon Dis 2008; 3: 31-44.

71 Bender MT, Niederman MS. Treatment guidelines for community-acquired pneumonia. Ann Res Hosp 2018; 2: 6.

72 Beghé $B$, Verduri $A$, Roca $M$, et al. Exacerbation of respiratory symptoms in COPD patients may not be exacerbations of COPD. Eur Respir J 2013; 41: 993-995.

73 Williams NP, Coombs NA, Johnson MJ, et al. Seasonality, risk factors and burden of community-acquired pneumonia in COPD patients: a population database study using linked health care records. Int J Chron Obstruct Pulmon Dis 2017; 12: 313-322.

74 Pavord ID, Lettis S, Anzueto A, et al. Blood eosinophil count and pneumonia risk in patients with chronic obstructive pulmonary disease: a patient-level meta-analysis. Lancet Respir Med 2016; 4: 731-741.

75 Halpin DMG, Criner GJ, Dransfield MT, et al. Triple versus dual combination therapy in chronic obstructive pulmonary disease in Asian countries: analysis of the IMPACT trial. Pulm Ther 2020; 7: 101-118.

76 Metlay JP, Kapoor WN, Fine MJ. Does this patient have community-acquired pneumonia? Diagnosing pneumonia by history and physical examination. JAMA 1997; 278: 1440-1445.

77 Lim WS, Baudouin SV, George RC, et al. BTS guidelines for the management of community acquired pneumonia in adults: update 2009. Thorax 2009; 64: Suppl. 3, iii1-iii55.

78 Tierney JF, Vale C, Riley R, et al. Individual participant data (IPD) meta-analyses of randomised controlled trials: guidance on their use. PLoS Med 2015; 12: e1001855. 